\title{
Two evolved supernova remnants with newly identified Fe-rich cores in the Large Magellanic Cloud ${ }^{\star}$
}

\author{
P. J. Kavanagh ${ }^{1}$, M. Sasaki ${ }^{1}$, L. M. Bozzetto ${ }^{2}$, S. D. Points ${ }^{3}$, E. J. Crawford ${ }^{2}$, J. Dickel ${ }^{4}$, \\ M. D. Filipović ${ }^{2}$, F. Haberl ${ }^{5}$, P. Maggi ${ }^{6}$, and E. T. Whelan ${ }^{1}$
}

\author{
${ }^{1}$ Institut für Astronomie und Astrophysik, Kepler Center for Astro and Particle Physics, Eberhard Karls Universität Tübingen, Sand 1, \\ 72076 Tübingen, Germany \\ e-mail: kavanagh@astro.uni-tuebingen.de \\ 2 Western Sydney University, Locked Bag 1797, Penrith South DC, NSW 1797, Australia \\ 3 Cerro Tololo Inter-American Observatory, Casilla 603, La Serena, Chile \\ ${ }^{4}$ Physics and Astronomy Department, University of New Mexico, MSC 07-4220, Albuquerque, NM 87131, USA \\ 5 Max-Planck-Institut für extraterrestrische Physik, Giessenbachstraße, 85748 Garching, Germany \\ ${ }^{6}$ Laboratoire AIM, CEA-IRFU/CNRS/Université Paris Diderot, Service d'Astrophysique, CEA Saclay, \\ 91191 Gif sur Yvette Cedex, France
}

Received 21 September 2015 / Accepted 29 October 2015

\section{ABSTRACT}

\begin{abstract}
Aims. We present a multi-wavelength analysis of the evolved supernova remnants MCSNR J0506-7025 and MCSNR J0527-7104 in the Large Magellanic Cloud.

Methods. We used observational data from XMM-Newton, the Australian Telescope Compact Array, and the Magellanic Cloud Emission Line Survey to study their broad-band emission and used Spitzer and H I data to gain a picture of the environment into which the remnants are expanding. We performed a multi-wavelength morphological study and detailed radio and X-ray spectral analyses to determine their physical characteristics.

Results. Both remnants were found to have bright X-ray cores, dominated by Fe L-shell emission, which is consistent with reverse shock-heated ejecta with determined Fe masses in agreement with Type Ia explosion yields. A soft X-ray shell, which is consistent with swept-up interstellar medium, was observed in MCSNR J0506-7025, suggestive of a remnant in the Sedov phase. Using the spectral fit results and the Sedov self-similar solution, we estimated the age of MCSNR J0506-7025 to be $\sim 16-28 \mathrm{kyr}$, with an initial explosion energy of $(0.07-0.84) \times 10^{51} \mathrm{erg}$. A soft shell was absent in MCSNR J0527-7104, with only ejecta emission visible in an extremely elongated morphology that extends beyond the optical shell. We suggest that the blast wave has broken out into a low density cavity, allowing the shock heated ejecta to escape. We find that the radio spectral index of MCSNR J0506-7025 is consistent with the standard -0.5 for supernova remnants. Radio polarisation at $6 \mathrm{~cm}$ indicates a higher degree of polarisation along the western front and at the eastern knot with a mean fractional polarisation across the remnant of $P \cong(20 \pm 6) \%$.

Conclusions. The detection of Fe-rich ejecta in the remnants suggests that both resulted from Type Ia explosions. The newly identified Fe-rich cores in MCSNR J0506-7025 and MCSNR J0527-7104 make them members of the expanding class of evolved Fe-rich remnants in the Magellanic Clouds.
\end{abstract}

Key words. ISM: supernova remnants - Magellanic Clouds - X-rays: ISM

\section{Introduction}

Supernovae are powerful stellar explosions that are important in many fields of astrophysics. They are key components of the engine that drives the physical and chemical evolution of the interstellar medium (ISM) in galaxies and are sources of cosmic rays (for a review, see Vink 2012). There are two types of supernovae. Core-collapse explosions signal the death of massive, short-lived stars $\left(\gtrsim 8 M_{\odot}\right)$. Type Ia supernovae are thought to result from the disruption or explosion of a carbon-oxygen (C-O) white dwarf surpassing the Chandrasekhar limit, either via accretion from a low mass stellar companion (the single degenerate scenario) or through the merger of two white dwarfs (the double degenerate scenario). However, it has been recently shown that Type Ia $\mathrm{SNe}$ may also result from the explosion of sub-Chandrasekhar white dwarfs (e.g., Sim et al. 2010; Woosley \& Kasen 2011). It is still

\footnotetext{
* Based on observations obtained with XMM-Newton, an ESA science mission with instruments and contributions directly funded by ESA Member States and NASA.
}

unclear as to which of the explosion channels is dominant in the Universe (Maoz 2008, and references therein).

Following the explosion, the freshly produced nucleosynthesis products are blown outwards, seeding the ISM of the host galaxy. The interaction of these expanding ejecta with the ambient medium creates a lasting imprint in the form of a supernova remnant (SNR). Recently, a new class of evolved remnants has been discovered in the Magellanic Clouds, the defining characteristic of which is the presence of an X-ray bright Fe-rich core, consistent with reverse shock-heated Type Ia ejecta (Borkowski et al. 2006). An additional feature of the Fe-rich plasma is that the gas is close to or in collisional ionisation equilibrium (CIE); i.e., the post-shock ionisation balance has been restored. This presents a problem for standard Type Ia models (e.g., Dwarkadas \& Chevalier 1998; Badenes et al. 2003, 2005), which predict that the central Fe-rich gas should be of low density and the ionisation timescales short, contrary to what is observed. Borkowski et al. (2006) argue that, to explain the higher-than-expected central densities, some pre-explosion seeding of the circumstellar 
medium (CSM) by the stellar progenitor must have occurred, with the best candidates being the so-called prompt single degenerate systems (Mannucci et al. 2006; Sullivan et al. 2006; Aubourg et al. 2008). These "prompt" events are produced by intermediate mass stars just below the core-collapse explosion limit ( 3.5-8 $M_{\odot}$, Aubourg et al. 2008) and are capable of enhancing the CSM density via ejection of the stellar envelope prior to the SN event. As stated by Borkowski et al. (2006), at later stages of the resulting SNR evolution, the Fe ejecta manifest as a dense Fe-rich gas close to the centre of the SNR.

Nine of these objects have been detected in the Large Magellanic Cloud (LMC) so far: 0454-67.2 (Seward et al. 2006), 0548-70.4 and 0534-69.9 (Hendrick et al. 2003), DEM L316A (Nishiuchi et al. 2001; Williams \& Chu 2005), DEM L238 and DEM L249 (Borkowski et al. 2006), MCSNR J0508-6902 (Bozzetto et al. 2014b), MCSNR J05086830, and MCSNR J0511-6759 (Maggi et al. 2014). In this paper we report on new X-ray observations and multi-wavelength studies of two known SNRs in the LMC, which we have found to exhibit bright $\mathrm{Fe}$-rich interiors and are therefore new additions to this relatively recent class of evolved SNRs.

Davies et al. (1976) used optical and radio data to identify many SNRs and candidate SNRs in the Magellanic Clouds. One of their candidate SNRs, LMC B0507-7029, was later confirmed by Filipovic et al. (1998) through cross-correlation of LMC radio and X-ray source catalogues. Haberl \& Pietsch (1999) reported on ROSAT Position Sensitive Proportional Counter (PSPC) observations of the LMC, labelling this source [HP99] 1139. While the PSPC source was confirmed as extended, its large off-axis angle of $\sim 49^{\prime}$ prevented a reliable SNR classification. Payne et al. (2008) later confirmed the SNR classification of this object using long-slit optical spectroscopy of the bright filaments. We hereafter refer to this object as MCSNR J0506-7025, following the Magellanic Cloud Emission Line Database ${ }^{1}$ (MCSNR) nomenclature, and the determined the position from our new X-ray data (see Sect. 5.1.1).

Haberl \& Pietsch (1999) also identified the ROSAT PSPC source [HP99] 1234, located next to the N 206 H II complex (Henize 1956) in the LMC. It was classified as a candidate SNR based on its X-ray hardness ratios and extent. An optical SNR candidate was identified at the location of [HP99] 1234 by Kavanagh et al. (2012) in a study of the N 206 superbubble using data from the Magellanic Cloud Emission Line Survey (MCELS, Smith et al. 2006), as shown in their Fig. 7. A more detailed analysis of this object using the ROSAT and MCELS data, supplemented with Molonglo Observatory Synthesis Telescope (MOST) radio continuum data, was performed by Kavanagh et al. (2013, hereafter KSP13), who confirmed its SNR nature. KSP13 suggested that MCSNR J0527-7104 is most likely in the Sedov phase of its evolution, determining an age of $\sim 25 \mathrm{kyr}$, though this is based on a somewhat uncertain ambient density estimate. Interestingly, only very faint $36 \mathrm{~cm}$ emission was detected from the remnant, with the emission well below the detection limit at all other frequencies, making it one of the weakest SNRs ever detected in the radio regime. Using the local stellar population and star formation history, KSP13 suggested a Type Ia explosion scenario for MCSNR J0527-7104, though a CC explosion due to a star "kicked" from N 206 could not be ruled out.

Our work on the multi-frequency studies of MCSNR J0506-7025 and MCSNR J0527-7104 using X-ray, radio, $\mathrm{HI}$, IR, and optical emission line data is arranged as

http://WWW.mcsnr.org/ follows: the observations and data reduction are described in Sect. 2; the radio analysis of MCSNR J0506-7025 and $\mathrm{X}$-ray analysis of both remnants are outlined in Sects. 3 and 4, respectively; results are given and discussed in Sect. 5; and finally we summarise our work in Sect. 6.

\section{Observations and data reduction}

\subsection{Optical}

The MCELS observations (Smith et al. 2006) were taken with the $0.6 \mathrm{~m}$ University of Michigan/Cerro Tololo Inter-American Observatory (CTIO) Curtis Schmidt Telescope equipped with a SITE $2048 \times 2048 \mathrm{CCD}$, producing individual images of $1.35^{\circ} \times 1.35^{\circ}$ at a scale of $2.3^{\prime \prime}$ pixel $^{-1}$. The survey mapped both the $\operatorname{LMC}\left(8^{\circ} \times 8^{\circ}\right)$ and the Small Magellanic Cloud $\left(3.5^{\circ} \times 4.5^{\circ}\right)$ in narrow bands covering [O III] $] \lambda 5007 \AA$, H $\alpha$, and [S II] 66716 , $6731 \AA$, in addition to matched green and red continuum bands. The survey data were flux calibrated and combined to produce mosaicked images. We extracted cutouts centred on MCSNR J0506-7025 from the MCELS mosaics. We subtracted the continuum images from the corresponding emission line images, thereby removing the stellar continuum and revealing the full extent of the faint diffuse emission. Finally, we divided the continuum subtracted [S II] image by the continuum subtracted $\mathrm{H} \alpha$ image to get a [S II]/H $\alpha$ map of MCSNR J0506-7025, with regions of $[\mathrm{S} \mathrm{II}] / \mathrm{H} \alpha>0.4$ indicative of the presence of an $\mathrm{SNR}$, or $[\mathrm{S} \mathrm{II}] / \mathrm{H} \alpha>0.67$ in the case of an SNR emission being contaminated by photoionisation from a nearby H II region (Mathewson \& Clarke 1973; Fesen et al. 1985), the latter being the case for MCSNR J0506-7025. We note that the MCELS observations of MCSNR J0527-7104 have already been reported in KSP13, though we use the images again here to aid in the multi-wavelength morphology analysis. The continuum subtracted emission line images for MCSNR J0506-7025 and MCSNR J0527-7104 are shown in Figs. 1-top right and 2-top right, respectively.

\subsection{Radio}

We observed MCSNR J0506-7025 with the Australian Telescope Compact Array (ATCA) on November 15 and 16 2011, using the new Compact Array Broadband Backend (CABB). The ATCA array configuration EW367 was used and observations were taken simultaneously at $\lambda=3$ and $6 \mathrm{~cm}(v=$ 9000 and $5500 \mathrm{MHz}$ ) using the dual-frequency mode. Baselines formed with the 6th ATCA antenna were excluded as the other five antennas were arranged in a compact configuration. The observations were carried out in the so called "snap-shot" mode, totalling $\sim 64 \mathrm{~min}$ of integration over a $14 \mathrm{~h}$ period. PKS B1934638 was used for flux density calibration ${ }^{2}$ and PKS B0530-727 was used for secondary (phase) calibration. The phase calibrator was observed twice every hour for a total 78 min over the whole observing session. The miriad ${ }^{3}$ (Sault et al. 1995) and karma (Gooch 1995) software packages were used for reduction and analysis. The $6 \mathrm{~cm}$ image (Fig. 3) has a resolution of $34^{\prime \prime} 7 \times 23^{\prime \prime}$. 1 at $\mathrm{PA}=44.4^{\circ}$, and an estimated $\mathrm{rms}$ noise of $0.2 \mathrm{mJy} / \mathrm{beam}$. Similarly, we made an image of MCSNR J0506-7025 at $3 \mathrm{~cm}$,

\footnotetext{
2 Flux densities were assumed to be $5.098 \mathrm{Jy}$ at $6 \mathrm{~cm}$ and $2.736 \mathrm{Jy}$ at $3 \mathrm{~cm}$.

3 http://www.atnf.csiro.au/computing/software/miriad/

4 http://www.atnf.csiro.au/computing/software/karma/
} 


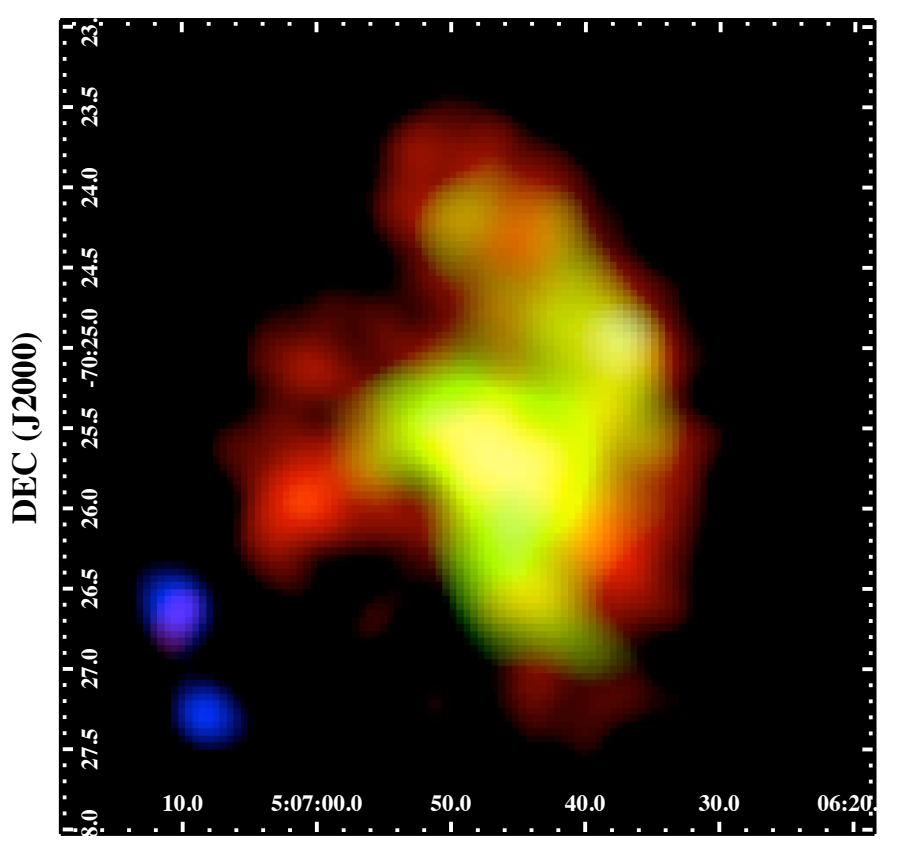

RA (J2000)

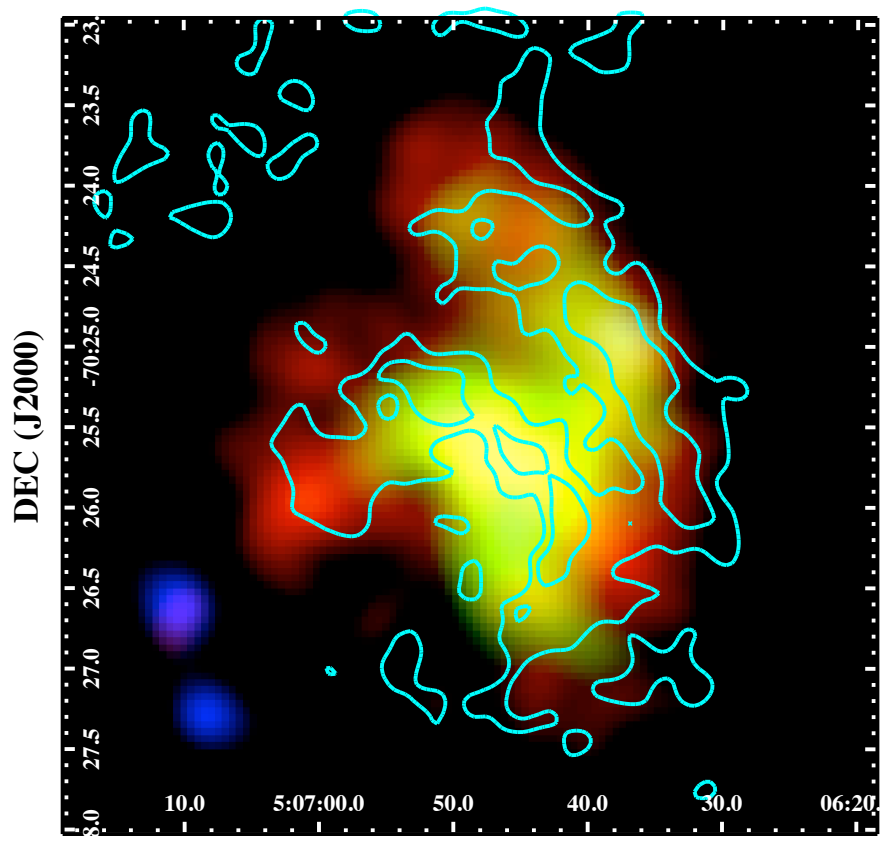

RA (J2000)

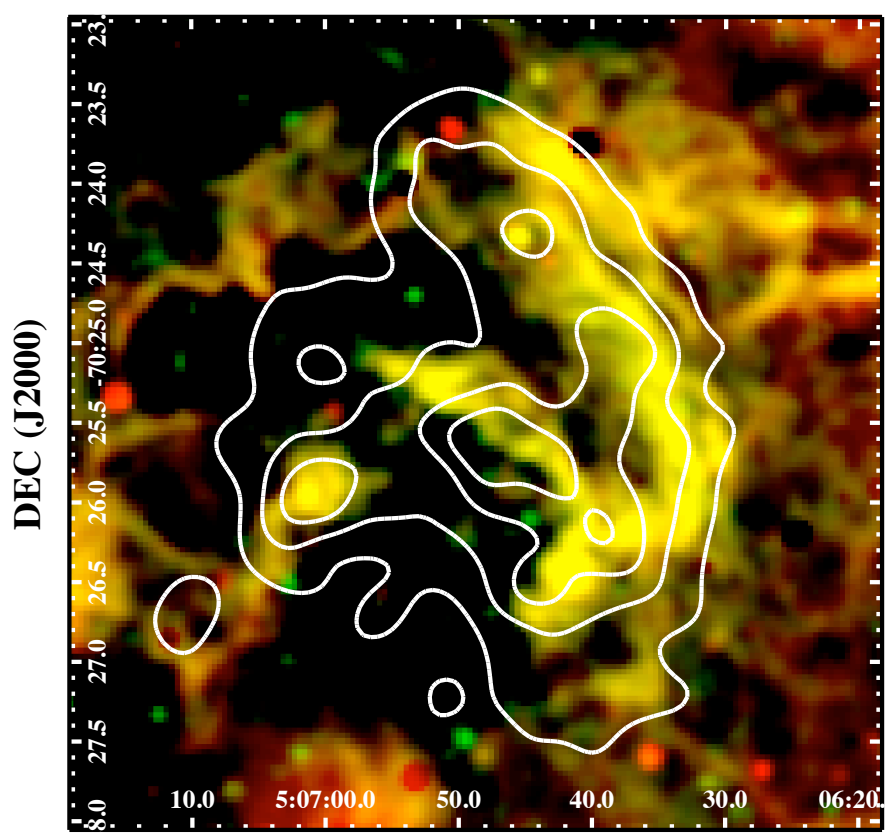

RA (J2000)

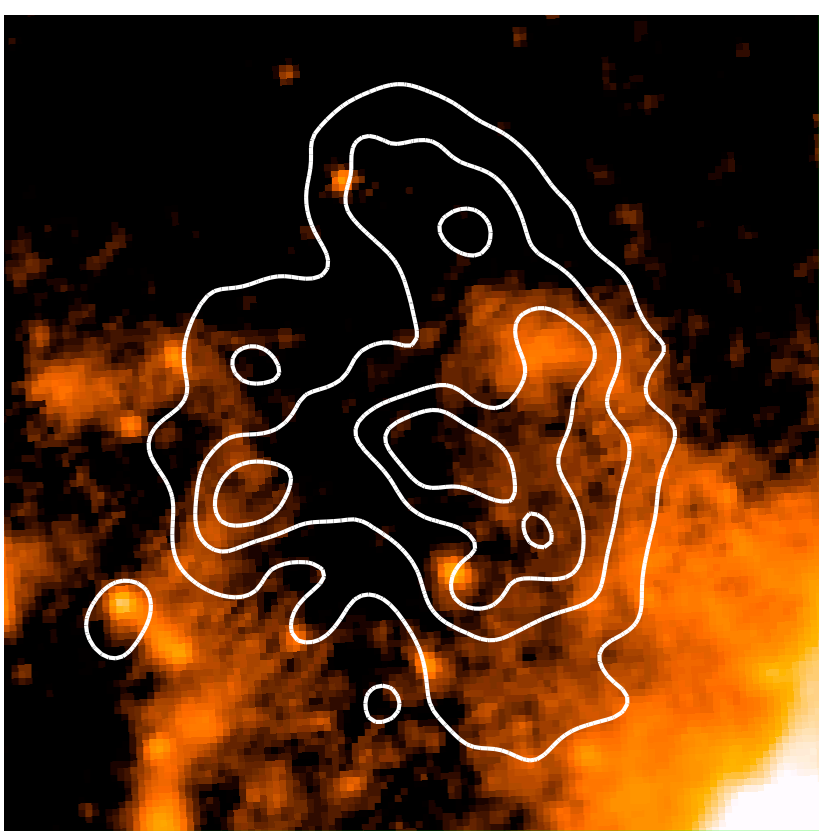

Fig. 1. Top left: XMM-Newton EPIC image of MCSNR J0506-7025 in false colour with RGB corresponding to 0.3-0.7 keV, 0.7-1.1 keV, and 1.1-4.2 keV, respectively. Top right: continuum subtracted MCELS image of MCSNR J0506-7025 with H $\alpha$ in red and [S II] in green overlaid with $0.3-0.7 \mathrm{keV}$ contours. The lowest contour level represents $3 \sigma$ above the average background surface brightness, with the remaining levels indicating $25 \%, 50 \%$, and $75 \%$ of the maximum above this level. Bottom left: same as top left but with [S II]/H $\alpha$ contours with the lowest level corresponding to $[\mathrm{S} \mathrm{II}] / \mathrm{H} \alpha=0.67$, and the remaining levels at $25 \%, 50 \%$, and $75 \%$ of the maximum above this level. Bottom right: Spitzer MIPS $24 \mu \mathrm{m}$ image of the MCSNR J0506-7025 region with soft X-ray contours from top right. The image scale is the same as in all other panels.

which has a resolution of $23^{\prime \prime} 7 \times 15^{\prime \prime} .3$ at $\mathrm{PA}=44.1^{\circ}$, and an estimated rms noise of $0.1 \mathrm{mJy} /$ beam.

In addition to these observations, we also make use of a $36 \mathrm{~cm}(843 \mathrm{MHz})$ MOST mosaic image (as described in Mills et al. 1984), a $20 \mathrm{~cm}(1377 \mathrm{MHz})$ mosaic image from Hughes et al. (2007) (the emission at both of the frequencies can be seen as superimposed contours in Fig. 3), and $6 \mathrm{~cm}$ and $3 \mathrm{~cm}$ mosaics (Dickel et al. 2010).

The analysis of radio emission from MCSNR J0527-7104 has already been reported in KSP13.

\subsection{X-ray}

MCSNR J0506-7025 and MCSNR J0527-7104 were observed by XMM-Newton (Jansen et al. 2001) on May 32013 (Obs. IDs 0720440201, PI M. Sasaki) and May 31-June 12014 (Obs. IDs 0741800101, PI P. J. Kavanagh), respectively. The primary instrument for the observations was the European Photon Imaging Camera (EPIC), which consists of a pn CCD (Strüder et al. 2001) and two MOS CCD imaging spectrometers (Turner et al. 2001). The observational data were reduced using the standard 


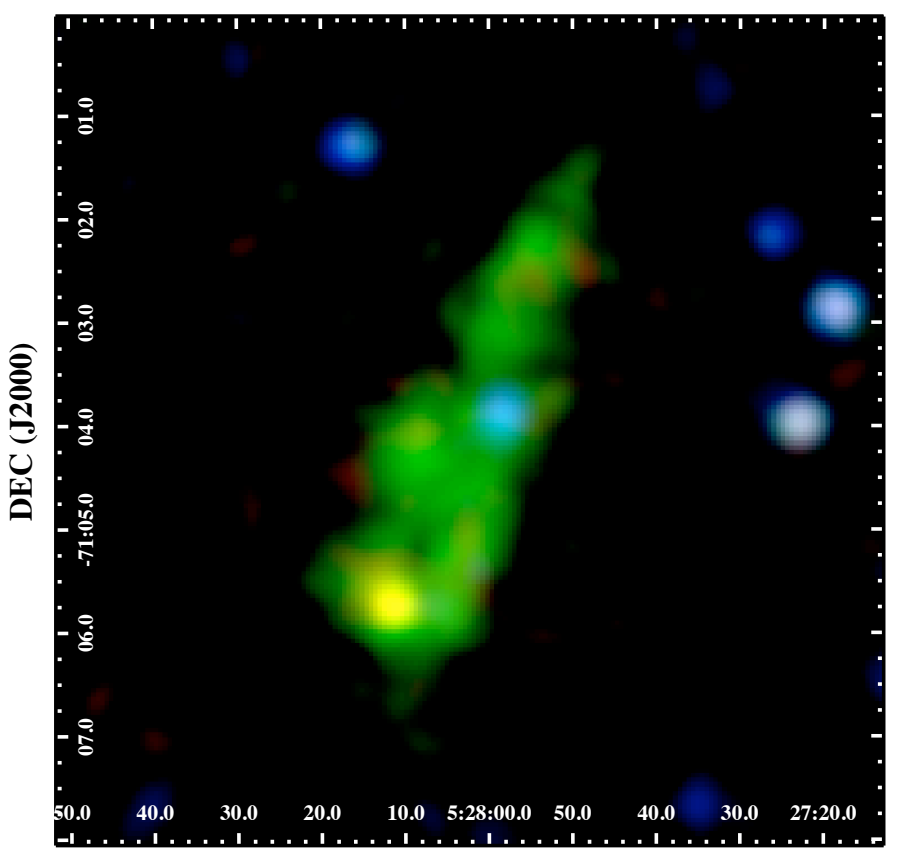

RA (J2000)

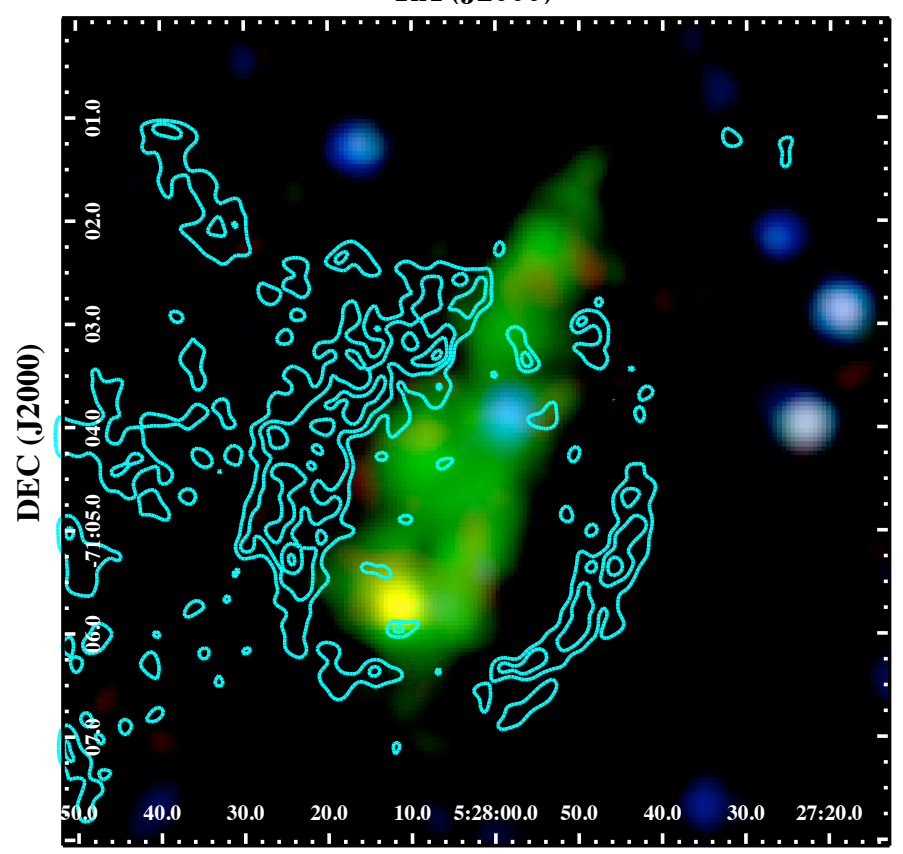

\section{RA (J2000)}

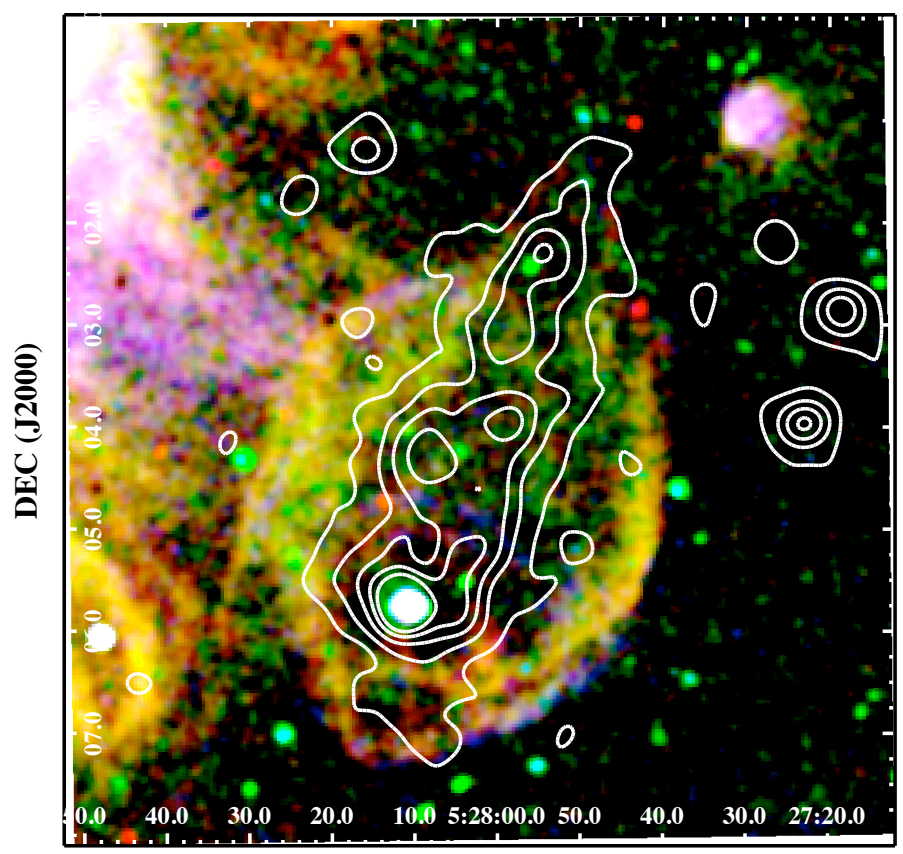

RA (J2000)

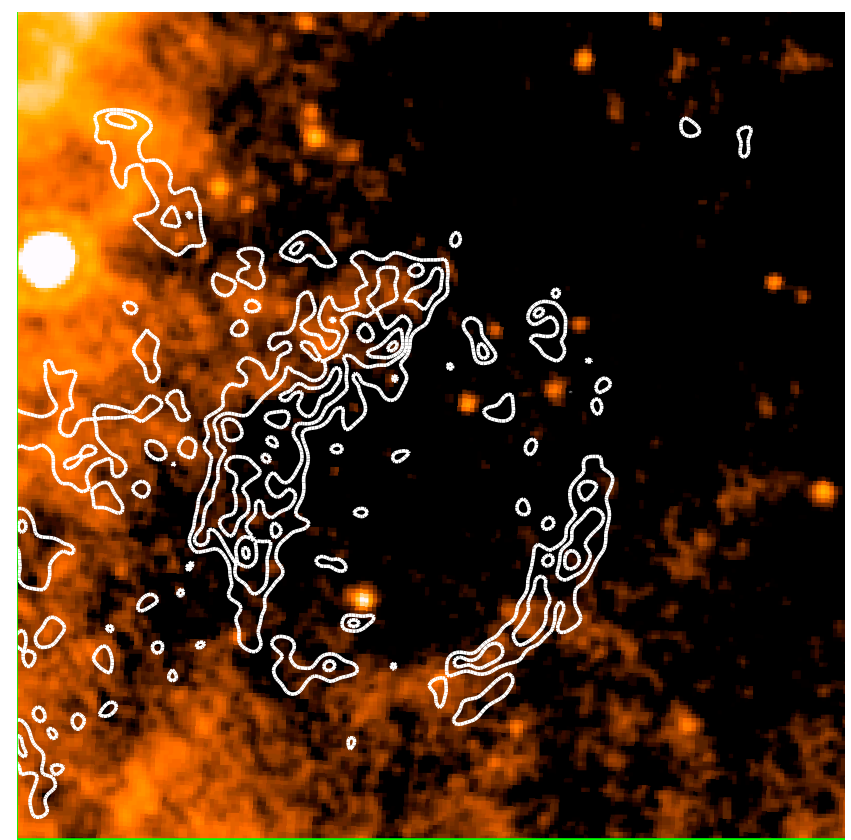

Fig. 2. Top left: XMM-Newton EPIC image of MCSNR J0527-7104 in false colour with RGB corresponding to 0.3-0.7 keV, 0.7-1.1 keV, and 1.1-4.2 keV, respectively. Top right: continuum subtracted MCELS image of MCSNR J0527-7104 from KSP13 with H $\alpha$ in red, [S II] in green, and [O III] in blue overlaid with $0.3-0.7 \mathrm{keV}$ contours. The lowest contour level represents $3 \sigma$ above the average background surface brightness, with the remaining levels indicating $25 \%, 50 \%$, and $75 \%$ of the maximum above this level. Bottom left: same as top left but with [S II]/H $\alpha$ contours with the lowest level corresponding to $[\mathrm{S} \mathrm{II}] / \mathrm{H} \alpha=0.67$, and the remaining levels at $25 \%, 50 \%$, and $75 \%$ of the maximum above this level (from KSP13). Bottom right: Spitzer MIPS $24 \mu \mathrm{m}$ image of the MCSNR J0527-7104 region with [S II]/H $\alpha$ contours from bottom left. The image scale is the same as in all other panels.

reduction tasks of $\mathrm{SAS}^{5}$ version 14.0.0, filtering for periods of high particle background. This resulted in $\sim 11 \mathrm{ks}$ for the EPIC-pn and $\sim 28 \mathrm{ks}$ each for the EPIC-MOS detectors for MCSNR J0506-7025, and $27 \mathrm{ks}$ for the EPIC-pn and $\sim 31 \mathrm{ks}$ each for the EPIC-MOS detectors for MCSNR J0527-7104, which were available for further analysis.

5 Science Analysis Software, see http://xmm.esac.esa.int/sas/

\subsection{Archival data}

\subsubsection{Infrared}

The cold environment surrounding the remnants can be revealed by infrared (IR) emission. To aid in the discussion of the morphology and environment of the remnant, we made use of data from the SAGE survey of the LMC (Meixner et al. 2006) with the Spitzer Space Telescope (Werner et al. 2004). During the 


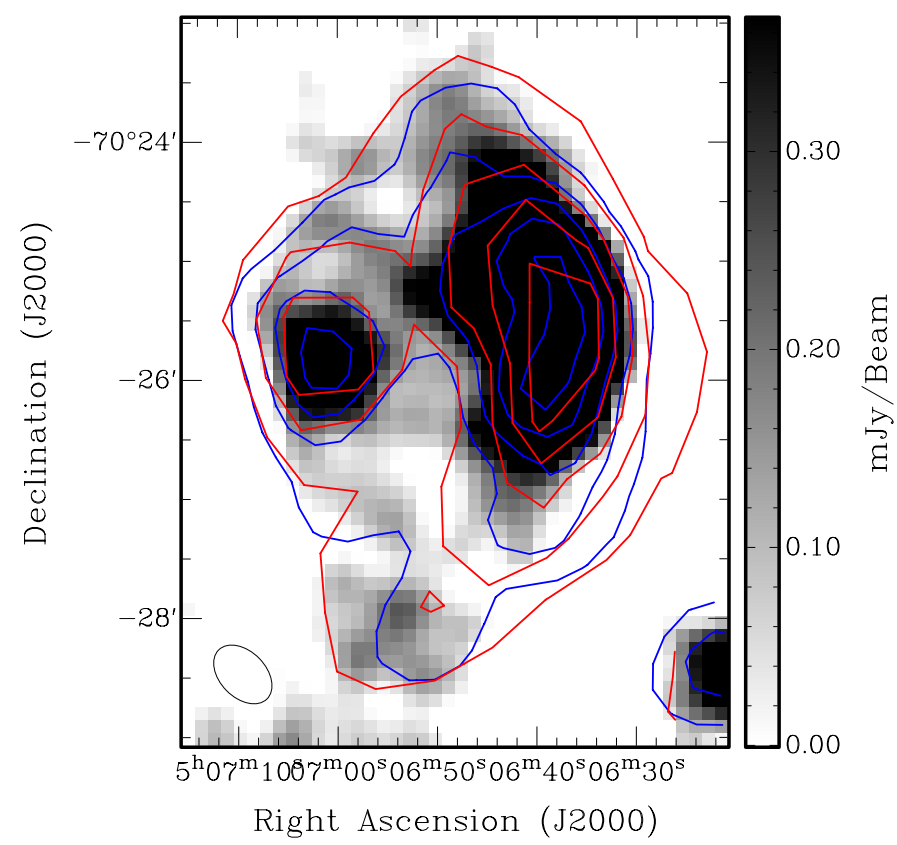

Fig. 3. ATCA $6 \mathrm{~cm}$ intensity image of MCSNR J0506-7025 overlaid with $36 \mathrm{~cm}$ contours (red; 1 to $11 \mathrm{mJy}$ in steps of $2 \mathrm{mJy}$ ) and $20 \mathrm{~cm}$ contours (blue; 1.8 to $6.6 \mathrm{mJy}$ in steps of $1.2 \mathrm{mJy}$ ). The ellipse in the lower left corner represents the synthesised beamwidth of $34.7 \times 23.1^{\prime \prime}$, and the sidebar shows the flux density scale and is given in mJy/Beam.

SAGE survey, a $7^{\circ} \times 7^{\circ}$ area of the LMC was observed with the Infrared Array Camera (IRAC, Fazio et al. 2004) in the $3.6 \mu \mathrm{m}, 4.5 \mu \mathrm{m}, 5.8 \mu \mathrm{m}$, and $8 \mu \mathrm{m}$ bands, and with the Multiband Imaging Photometer (MIPS, Rieke et al. 2004) in the $24 \mu \mathrm{m}$, $70 \mu \mathrm{m}$, and $160 \mu \mathrm{m}$ bands. The MIPS $24 \mu \mathrm{m}$ images provide us with a picture of the stochastically and thermally heated dust in the region of MCSNR J0506-7025 and MCSNR J0527-7104, with spatial resolution comparable to XMM-Newton, to give an indication of the distribution of cool material. We obtained the $24 \mu \mathrm{m}$ MIPS mosaicked, flux-calibrated (in units of $\mathrm{MJy} \mathrm{sr}^{-1}$ ) images processed by the SAGE team from the NASA/IPAC Infrared Science Archive ${ }^{6}$. The pixel sizes correspond to $4.8^{\prime \prime}$ for the $24 \mu \mathrm{m}$ band, $\sim 1.2 \mathrm{pc}$ at the LMC distance.

\subsection{2. $\mathrm{HI}$}

To gain an understanding of the neutral $\mathrm{H}$ in the regions of MCSNR J0506-7025 and MCSNR J0527-7104 we used data from the HI survey of the LMC by the ATCA and Parkes facilities $^{7}$, which are described in detail by Staveley-Smith et al. (2003) and Kim et al. (2003).

\section{Radio analysis of MCSNR J0506-7025}

\subsection{Radio spectral index}

To estimate the spectral index of MCSNR J0506-7025, three different methods were used. The first is a simple fit to the total integrated flux densities taken at the different wavelengths. The second, $\alpha_{\mathrm{TT}}$, uses the temperature - temperature (T-T) plot method which has the benefit that it is immune to the zero-level in the individual maps. The third, $\alpha_{S_{\text {med }}}$, takes the median value

\footnotetext{
6 See http://irsa.ipac. caltech.edu/data/SPITZER/SAGE/

7 http://www.atnf.csiro.au/research/HI/mc/queryForm. html
}

of a spectral index map. For all three methods, unless otherwise stated, the data at each wavelength were convolved down to the lowest resolution $\left(36 \mathrm{~cm}\right.$; beam size $=46$.' $4 \times 43^{\prime \prime}$. 0 ) to make the images comparable.

Integrated flux density measurements were taken at $36 \mathrm{~cm}$ (80.1 mJy), $20 \mathrm{~cm}$ (76.4 mJy), and $6 \mathrm{~cm}$ (50.6 mJy). Uncertainties in these flux density measurements predominately arose from defining the edge of the remnant. We estimate that the errors in all flux density estimates are within $10 \%$. The integrated flux density measurement at $3 \mathrm{~cm}$ is a little more problematic due to contamination from the faint $\mathrm{H}$ II region to the west of the SNR. We estimated the flux density at $3 \mathrm{~cm}$ to be $46( \pm 15) \mathrm{mJy}$. The resulting plot and fit are given in Fig. 5, where $\alpha_{\mathrm{S}}=-0.25 \pm 0.02$. This is relatively flat compared to the typical SNR spectral index of $\sim-0.5$.

To verify the spectral index of the emission, a spectral map was created only between 20 and $6 \mathrm{~cm}$ wavelengths. The $36 \mathrm{~cm}$ image was not used in this scenario as the missing short spacings responsible for the large scale emission severely impacted the flux density of the image, resulting in non-sensical values of $\alpha$. The spectral index map was created by reprocessing both sets of data to a common $u-v$ range, and then fitting $S \propto v^{\alpha}$ pixel by pixel using both images, and is shown in Fig. 4-left. The resulting spectral pixel values were binned, and a histogram of their distribution is shown in Fig. 4-right. The 20 and $6 \mathrm{~cm}$ images were masked at levels of 2.0 and $1.8 \mathrm{mJy}$, respectively. Values that fell below these cutoffs were blanked from the output image. The resulting median spectral index from this data is $\alpha_{S_{\text {med }}}=-0.33$.

We also estimated the spectral index using the T-T plot method (Costain 1960; Turtle et al. 1962), i.e., the spectral index is determined by finding the scaling factor which gives the best agreement between temperature curves at given frequencies. We created T-T plots between $36-20 \mathrm{~cm}, 36-6 \mathrm{~cm}$, and $20-6 \mathrm{~cm}$ (see Fig. 6). This method is seen as the most reliable as it neglects any external flux (e.g., background emission). The resulting spectral indices, $-0.64,-0.47,-0.36$, show the non-thermal nature of the emission. Taking the mean spectral index of the three plots results in $\alpha=-0.49$, in line with the index characteristic of SNRs of -0.5 .

Although there is clearly some variation in values found from the various methods and images used, all the spectral index results point to the emission being non-thermal in nature. The most reliable results, the T-T plot method, showed a spectral index inline with the standard -0.5. If we do not take the other results into account (i.e., the flatter spectra from analyses such as the spectral map between 20 and $6 \mathrm{~cm}$ ), we are left with a spectral index that is characteristic of an evolved remnant.

\subsection{Magnetic field}

A polarisation image of MCSNR J0506-7025 was created at $6 \mathrm{~cm}$ using the miriad task impol, from $Q$ and $U$ Stokes parameters. The mean fractional polarisation was calculated using flux density and polarisation:

$P=\frac{\sqrt{S_{Q}^{2}+S_{U}^{2}}}{S_{I}}$,

where $S_{Q}, S_{U}$, and $S_{I}$ are integrated intensities for the $Q, U$, and $I$ Stokes parameters. A signal-to-noise cut-off of $2 \sigma$ was used for the $Q$ and $U$ images, and a level of $8 \sigma$ for the intensity image. Values that fell below these cut-off levels are blanked in the output image. Additionally, the fractional polarisation map 

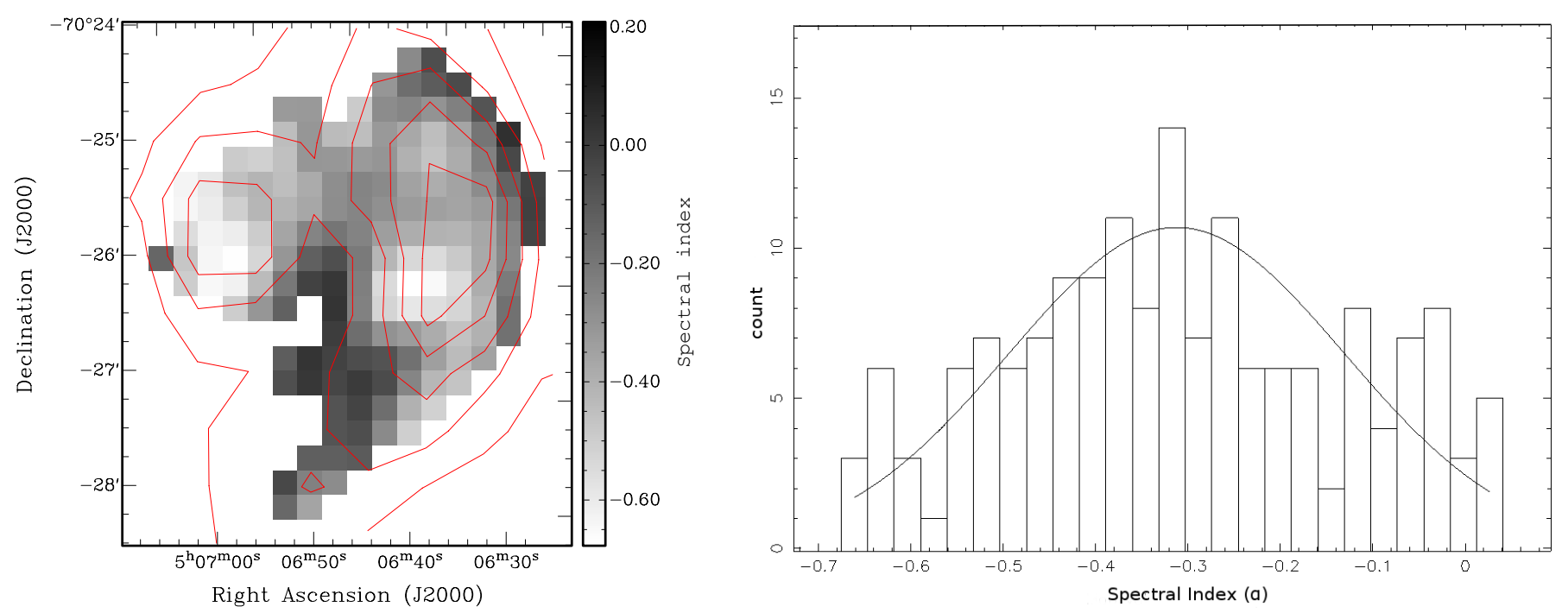

Fig. 4. Left: spectral index map between 20 and $6 \mathrm{~cm}$ for MCSNR J0506-7025. The contours are the same $36 \mathrm{~cm}$ contours as shown in Fig. 3 . Right: histogram of the distribution of spectral index values from the spectral map in left.

Table 1. Spectral index estimates of MCSNR J0506-7025.

\begin{tabular}{cccccc}
\hline \hline$\alpha_{\mathrm{S}}$ & $\alpha_{\mathrm{S}}$ & $\alpha_{\mathrm{TT}}$ & $\alpha_{\mathrm{TT}}$ & $\alpha_{\mathrm{TT}}$ & $\alpha_{\mathrm{S}_{\text {med }}}$ \\
$(36-20-6-3 \mathrm{~cm})$ & $(20-6-3 \mathrm{~cm})$ & $(36-20 \mathrm{~cm})$ & $(36-6 \mathrm{~cm})$ & $(20-6 \mathrm{~cm})$ & $(20-6 \mathrm{~cm})$ \\
\hline$-0.25 \pm 0.02$ & $-0.28 \pm 0.02$ & -0.64 & -0.47 & -0.36 & -0.33 \\
\hline
\end{tabular}

Notes. Columns 1 and 2: spectral indices from direct integrated flux density measurements at the wavelengths given in parenthesis. Columns 3-5: spectral indices resulting from T-T plots using the images given in parenthesis. Column 6: median value of the spectral indices taken from a spectral map at a resolution of $20 \mathrm{~cm}$.

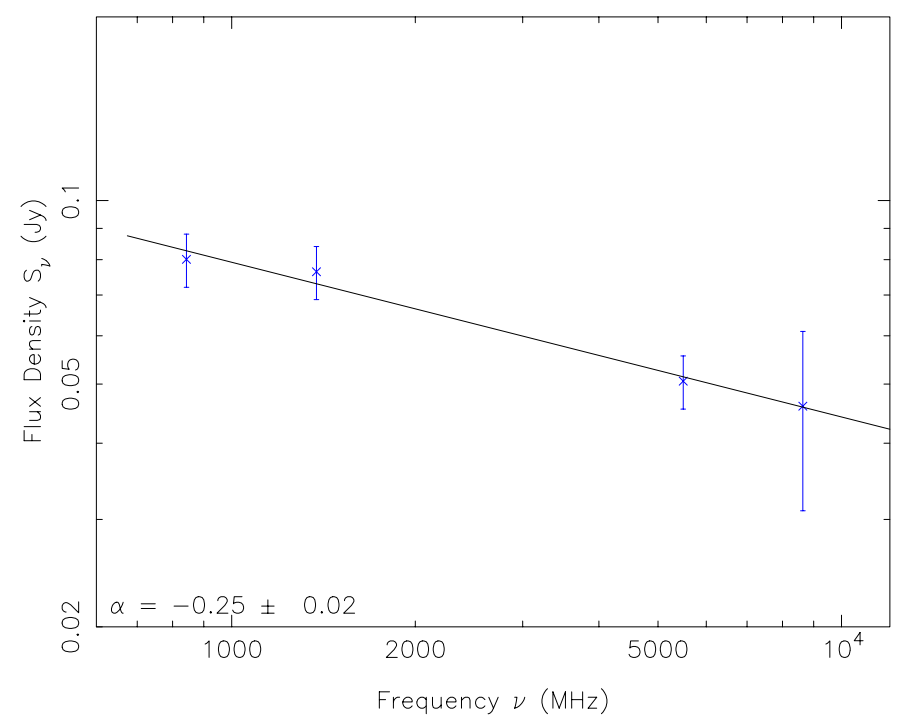

Fig. 5. Spectral index fit to MCSNR J0506-7025 using integrated flux density measurements at $36,20,6$, and $3 \mathrm{~cm}$.

$p$ (shown in Fig. 7) was compared with its associated error map $\sigma_{p}$, and pixels were blanked for $p / \sigma_{p} \leq 2$. The emission in the west shows clear amplification of the magnetic field toward the shock front, with values of $P \approx 30 \%$. Similarly, the emission in the east shows this amplification, where fractional polarisation values reach as high as $45 \%$.

Mean polarisation across the remnant at $6 \mathrm{~cm}$ was found to be $P \cong(20 \pm 6) \%$. As there are two distinct regions of this SNR, we have taken independent measurements for both. We find a mean polarisation of $P \cong(19 \pm 6) \%$ for the SNR emission to the west, and a slightly higher $P \cong(24 \pm 7) \%$ for the emission in the east. The orientation of the magnetic field vectors can be seen in Fig. 8. The length of the vectors have been halved for display purposes.

In comparison to other LMC SNRs arising from a Type Ia SNe, MCSNR J0506-7025 shows similar mean levels of fractional polarisation: MCSNR J0509-6731 with $26 \pm 13 \%$ at $6 \mathrm{~cm}$ (Bozzetto et al. 2014a); MCSNR J0533-7202 with $12 \pm$ 7\% (Bozzetto et al. 2013); MCSNR J0536-7038 with $35 \pm 8 \%$ (Bozzetto \& Filipović 2014); while MCSNR J0519-6902 exhibited a much lower mean fractional polarisation at a level of 2.2 and $3.2 \%$ at 6 and $3 \mathrm{~cm}$, respectively (Bozzetto et al. 2012).

\section{X-ray analysis}

\subsection{X-ray imaging}

We produced images and exposure maps in various energy bands from the flare-filtered event lists for each EPIC instrument in each observation. We filtered for single and double-pixel events (PATTERN $\lesssim 4$ ) from the EPIC-pn detector, with only singlepixel events considered below $0.5 \mathrm{keV}$ to avoid the higher detector noise contribution from the double-pixel events at these energies. All single to quadruple-pixel events (PATTERN $\lesssim 12$ ) were considered for the MOS detectors.

We used three energy bands suited to the analysis of the spectra of SNRs. A soft band from $0.3-0.7 \mathrm{keV}$ includes strong lines from $\mathrm{O}$; a medium band from $0.7-1.1 \mathrm{keV}$ comprises Fe L-shell lines as well as $\mathrm{Ne} \operatorname{He} \alpha$ and $\mathrm{Ly} \alpha$ lines; and a hard band (1.1$4.2 \mathrm{keV}$ ) which includes lines from $\mathrm{Mg}, \mathrm{Si}, \mathrm{S}, \mathrm{Ca}$, and $\mathrm{Ar}$. 

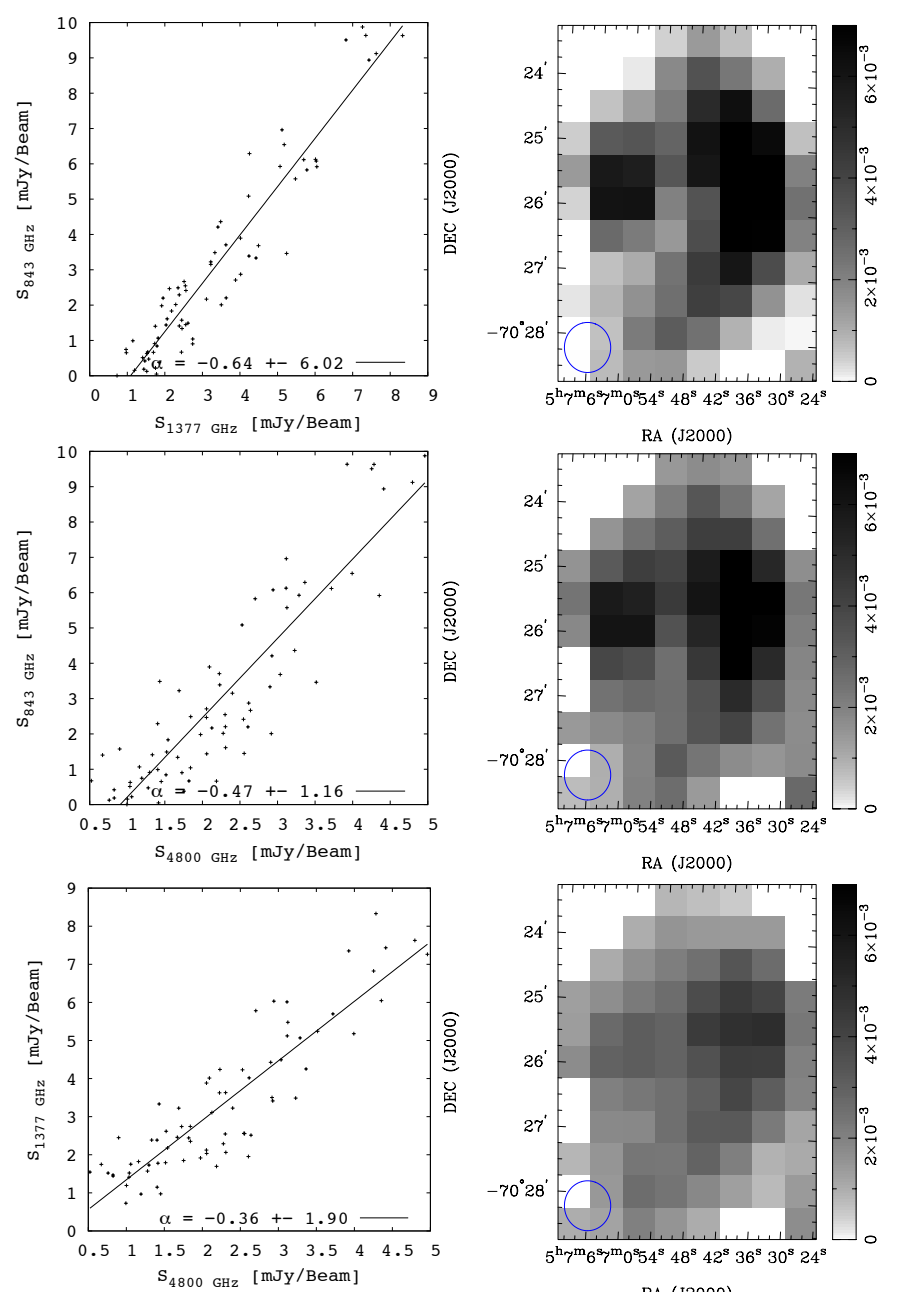

Fig. 6. Left: T-T plots of MCSNR J0506-7025 between 36-20 cm, 36$6 \mathrm{~cm}$, and $20-6 \mathrm{~cm}$ (top to bottom). Right: intensity images showing the region and strength of the emission from 36,20 , and $6 \mathrm{~cm}$ images (top-to-bottom).

We subtracted the detector background from the images using filter-wheel-closed data (FWC). The contribution of the detector background to each EPIC detector was estimated from the count rates in the corners of the images, which were not exposed to the sky. We then subtracted appropriately-scaled FWC data from the raw images. We merged the EPIC-pn and EPIC-MOS images from each observation into combined EPIC images and performed adaptive smoothing of each using a template determined from the combined energy band (0.3-4.2 keV) EPIC image. The sizes of Gaussian kernels were computed at each position in order to reach a signal-to-noise ratio of five, setting the minimum full width at half maximum of the kernels to $\sim 8^{\prime \prime}$. The smoothed images were then divided by the corresponding vignetted exposure maps. Finally, we produced three-colour images of MCSNR J0506-7025 and MCSNR J0527-7104 which are shown in Figs. 1-top left and 2-top left, respectively.

\subsection{Point sources}

We identified point sources in and around each SNR using the SAS task edetect_chain. Images were extracted from each of the EPIC instruments in the standard energy bands $0.2-$ $0.5 \mathrm{keV}, 0.5-1 \mathrm{keV}, 1-2 \mathrm{keV}, 2-4.5 \mathrm{keV}$, and 4.5-12 keV, with

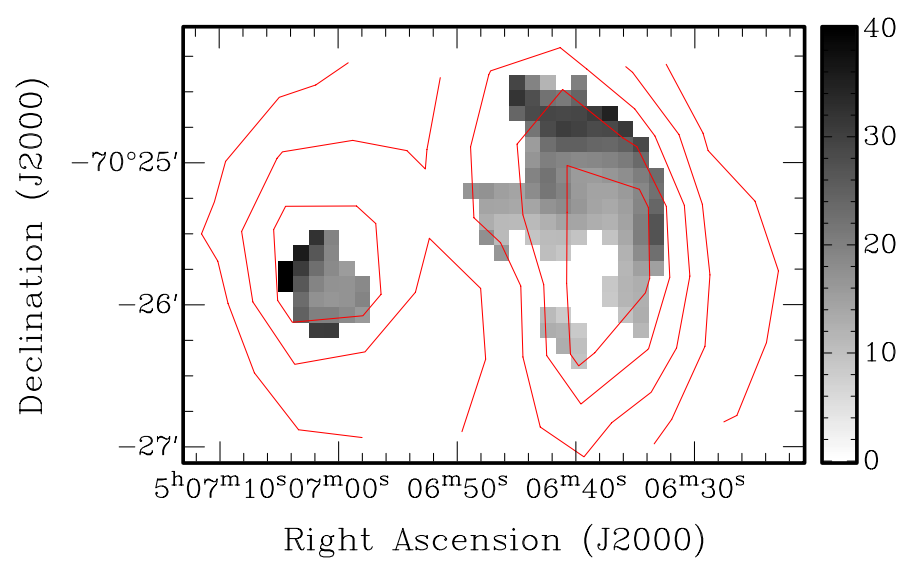

Fig. 7. Fractional polarisation ( $p$ ) of MCSNR J0506-7025 at $6 \mathrm{~cm}$. The side bar quantifies the level of fractional polarisation in percent. Pixels where the error was half the measured fractional polarisation or greater were blanked from the image. The contours are the same $36 \mathrm{~cm}$ contours as shown in Fig. 3.

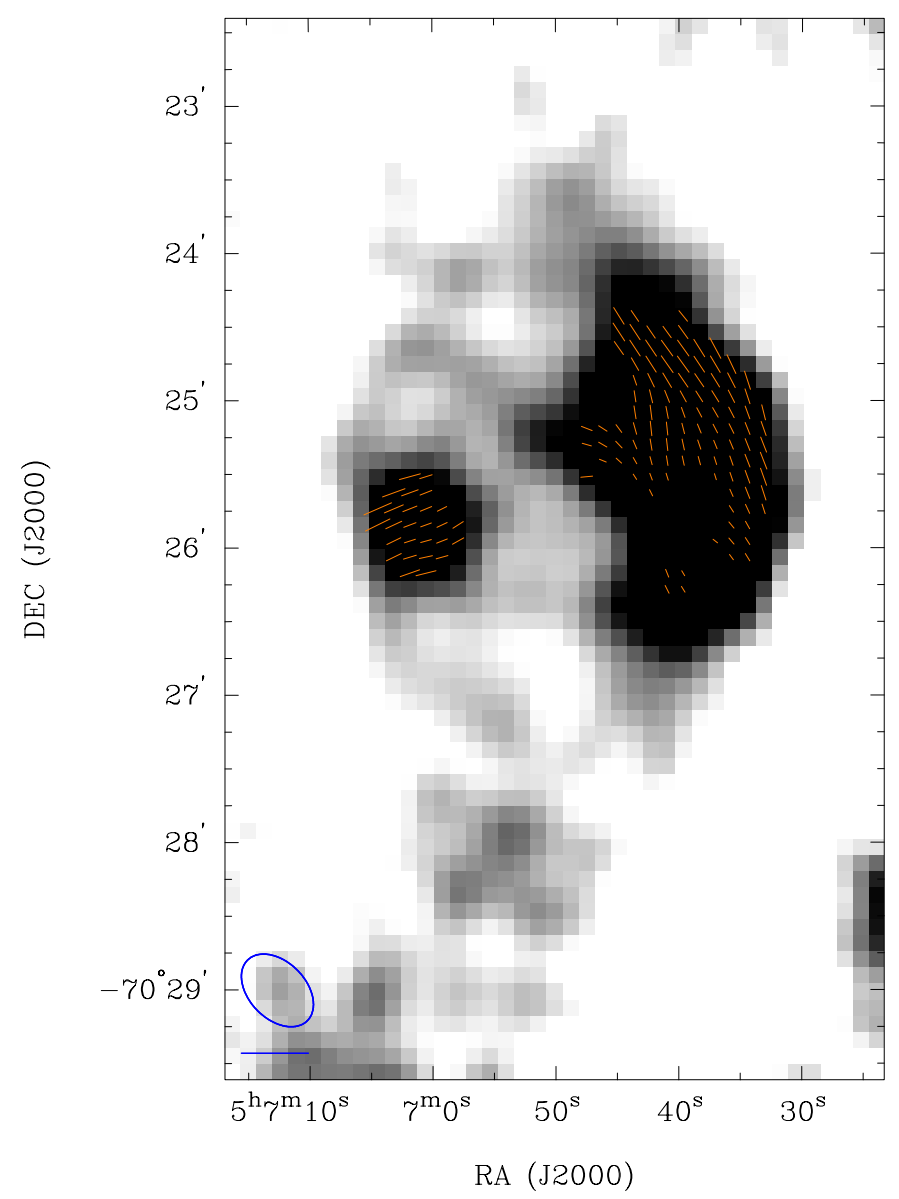

Fig. 8. Magnetic field line vectors of MCSNR J0506-7025 from the ATCA $6 \mathrm{~cm}$ observations overlaid on an intensity image at the same wavelength. The ellipse in the lower left corner represents the synthesised beamwidth of $34.7 \times 23.1^{\prime \prime}$.

the same pattern filtering criteria outlined in Sect. 4.1. False detections due to the extended emission of the SNRs were removed after visual inspection. No point sources were detected within the dimensions of MCSNR J0506-7025. Two sources were found projected against MCSNR J0527-7104: foreground star HD 36877, the bright source located in the southeast of the 
elongated remnant emission; and a source with J2000 coordinates of RA $=05^{\mathrm{h}} 27^{\mathrm{m}} 58.47^{\mathrm{s}}$ and Dec $=-71^{\mathrm{d}} 03^{\mathrm{m}} 53.7^{\mathrm{s}}$, the hard source near the western edge to which we assign the identifier XMMU J052758.4-710353. Given the hard nature of its emission, XMMU J052758.4-710353 is most likely a background AGN.

\subsection{X-ray spectral analysis}

For the spectral analysis, we made use of the EPIC-pn and EPICMOS data. Although less sensitive, the EPIC-MOS spectral resolution is slightly better than the EPIC-pn, and therefore helps to constrain the parameters of the spectral models. Before proceeding with the spectral extraction we generated vignettingweighted event lists for each EPIC instrument to correct for the effective area variation across our extended source using the SAS task evigweight. Spectra were extracted from elliptical regions encompassing the X-ray extent of the SNRs. Backgrounds were extracted from nearby source and diffuse emission free regions.

We determined exclusion radii for contaminating point sources (see Sect. 4.2) using the contour method of the SAS task region. The task calculates the point spread function (PSF) at the source position and normalises for the source brightness. Source counts were then removed down to a PSF threshold of 0.2 times the local background, that is, the point source is excluded down to a level where the surface brightness of the point source is $20 \%$ of the background. This method has the advantage that the point source exclusion regions follow the brightness of the source, so that brighter sources have larger exclusion regions and shapes corresponding approximately to the source PSF. Hence, the number of diffuse counts is maximised by tailoring the point source exclusion regions to the individual sources. We determined exclusion radii of $\sim 35^{\prime \prime}$ and $\sim 18^{\prime \prime}$ for HD 36877 and XMMU J052758.4-710353, respectively.

All spectra were rebinned so that each bin contained a minimum of 30 counts to allow the use of the $\chi^{2}$ statistic during spectral fitting. The EPIC-pn and EPIC-MOS source and background spectra from each observation were fitted simultaneously using XSPEC (Arnaud 1996) version 12.8.2p with abundance tables set to those of Wilms et al. (2000), photoelectric absorption cross-sections set to those of Balucinska-Church \& McCammon (1992), and atomic data from ATOMDB 3.0.1 ${ }^{8}$ with the latest equilibrium and non-equilibrium data.

\subsubsection{X-ray background}

A comprehensive description of the X-ray background constituents and spectral modelling can be found in Maggi et al. (2016). Here we briefly summarise the treatment of the X-ray background in the cases of MCSNR J0506-7025 and MCSNR J0527-7104.

The X-ray background consists of the astrophysical X-ray background (AXB) and particle induced background. The AXB typically comprises four or fewer components (Snowden et al. 2008; Kuntz \& Snowden 2010), namely the unabsorbed thermal emission from the Local Hot Bubble, absorbed cool and hot thermal emission from the Galactic halo, and an absorbed power law representing unresolved background active galactic nuclei (AGN). The spectral properties of the background AGN component were fixed to the well known values of $\Gamma \sim 1.46$ and a normalisation equivalent to 10.5 photons $\mathrm{cm}^{-2} \mathrm{~s}^{-1} \mathrm{sr}^{-1}$ at $1 \mathrm{keV}$ (Chen et al. 1997). The foreground

\footnotetext{
8 http://www . atomdb.org/index.php
}

absorbing material comprises both Galactic and LMC components. The foreground Galactic absorption component was fixed at $7.9 \times 10^{20} \mathrm{~cm}^{-2}$ for MCSNR J0506-7025 and $6.9 \times 10^{20} \mathrm{~cm}^{-2}$ for MCSNR J0527-7104, based on the Dickey \& Lockman (1990) HI maps, determined using the HEASARC $N_{\mathrm{H}}$ Tool ${ }^{9}$. The foreground LMC absorption component, with abundances set to those of the LMC, was allowed to vary.

The particle-induced background of the EPIC consists of the quiescent particle background (QPB), instrumental fluorescence lines, electronic read-out noise, and residual soft proton (SP) contamination. To determine the contribution of these components we made use of vignetting corrected FWC data. We extracted FWC spectra from the same detector regions as the observational source and background spectra. The EPIC-pn and EPIC-MOS FWC spectra were fitted with the empirical models developed by Sturm (2012) and Maggi et al. (2016), respectively. Since these spectral components are not subject to the instrumental response, we used a diagonal response in XSPEC. The resulting best-fit model was included and frozen in the fits to the observational spectra, with only the widths and normalisations of the strongest fluorescence lines allowed to vary ${ }^{10}$. We also included a multiplicative constant to normalise the continuum to the observational spectra using the high energy tail $(E>5 \mathrm{keV}$ ) where the QPB component dominates. The residual SP contamination was fitted by a power law not convolved with the instrumental response (Kuntz \& Snowden 2008). The residual SP component was only required in the spectral analysis of MCSNR J0506-7025.

\subsubsection{Spectral fitting: MCSNR J0506-7025}

The X-ray emission of MCSNR J0506-7025 consists of a centrally bright region peaking in the $0.7-1.1 \mathrm{keV}$ range, surrounded by a softer shell (see Fig. 1-top left). A similar morphology has been observed in other LMC remnants and is discussed in detail in Sect. 5.1.1. The centrally peaked $0.7-1.1 \mathrm{keV}$ emission suggests that the Fe L-shell complex dominates at these energies. Inspection of the spectrum confirmed this to be the case with an obvious Fe L-shell "bump" present. The softer outer emission is most likely due to a swept-up and shock-heated ISM shell, typical of evolved SNRs. The separation of core and shell regions is not straightforward. As seen in Fig. 1-top left, the 0.7-1.1 keV emission is not confined to a central well-defined core, but rather is somewhat smeared out from the core in both northernly and southernly directions. It is not clear if this emission is due to $\mathrm{Fe}$ or is simply caused by enhancements in the shell emission. The latter may be the case in the northern extension from the core, which is correlated with bright soft emission. However, in the southern extension the $0.7-1.1 \mathrm{keV}$ emission is anti-correlated with the soft shell. Since we cannot define clear core and shell regions, we decided to fit the spectrum of the entire SNR, incorporating both shell and core components. We note here that we also tried to incorporate additional pure heavy element plasmas such as $\mathrm{O}$ to account for ejecta emission apart from Fe. However, in fits to the spectrum of the entire remnant and the test spectra extracted from the brightest core regions, additional ejecta components were not required with only upper limits determined.

\footnotetext{
9 http://heasarc.gsfc.nasa.gov/cgi-bin/Tools/w3nh/ w3nh.pl

${ }^{10}$ Following the method outlined in the XMM-Newton Extended Source Analysis Software Cookbook available at http://heasarc.gsfc. nasa.gov/docs/xmm/xmmhp_xmmesas.html
} 
Therefore, we consider only the Fe ejecta component in the forthcoming analysis.

To account for the shell emission, we included a thermal plasma model in the spectral fits absorbed by foreground Galactic and LMC material. We made use of a non-equilibrium ionisation (NEI) model in XSPEC, appropriate for SNRs, namely the plane-parallel vpshock model (Borkowski et al. 2001). The vpshock model features a linear distribution of ionisation ages behind the shock. Given the large size of the MCSNR J0506-7025 (see Sect. 5.1.1), we assumed that the remnant is in the Sedov phase and the emission from the soft $\mathrm{X}$-ray shell is dominated by swept-up ISM. Therefore, we also fit the spectrum with the Sedov dynamical model, implemented as vsedov in XSPEC (Borkowski et al. 2001).

To account for the central Fe L-shell emission we used a pure Fe thermal plasma model, achieved by setting all abundances apart from Fe to 0 . We performed trial fits with CIE models and NEI models. We note that for the CIE fits we did not use the standard vapec model. The reason for this is because it not possible to set the $\mathrm{H}$ abundance to 0 in the vapec model. In past versions of XSPEC, this was not a problem as the abundance of Fe could be set sufficiently high to render any contribution of $\mathrm{H}$ insignificant. However, in the most recent versions of XSPEC, the abundance of elements in the vapec model has a much lower maximum limit and the effect of $\mathrm{H}$ on the spectral fits can no longer be ignored. To circumvent this issue, we used the NEI model vnei and froze the ionisation parameter to $1 \times 10^{12} \mathrm{~s} \mathrm{~cm}^{-3}$, consistent with a plasma in CIE (Smith \& Hughes 2010). The vnei model does allow the value abundance of $\mathrm{H}$ to be set to 0 , therefore providing a pure Fe plasma model. The CIE fits produced a good fit to the core Fe emission. Similarly, the ionisation parameters in the NEI fits were consistently $\gtrsim 10^{12} \mathrm{~s} \mathrm{~cm}^{-3}$, indicative of a CIE plasma. Therefore, we adopted the CIE model to account for the Fe L-shell emission.

Both the vpshock+vnei and vsedov+vnei models provided good fits to the MCSNR J0506-7025 spectrum with reduced $\chi^{2}\left(\chi_{v}^{2}\right)$ of 1.16 and 1.11 respectively. The best-fit parameters for both models are given in Table 2, with the best-fit vsedov+vnei spectrum shown in Fig. 9-left. These fit results are discussed in Sect. 5.1.2.

\subsubsection{Spectral fitting: MCSNR J0527-7104}

The X-ray morphology of MCSNR J0527-7104 is extremely unusual (see Sect. 5.2.1 for a thorough discussion) with the emission almost exclusively confined to the $0.7-1.1 \mathrm{keV}$ band (see Fig. 2-top left), suggesting that the spectrum could be dominated by emission lines from the Fe L-shell complex, which was confirmed upon inspection of the spectrum. There is no evidence of soft X-ray emission filling out the optical shell of MCSNR J0527-7104 (see Fig. 2-bottom left). We therefore proceeded by applying a single thermal component consisting of pure Fe. Initial trial fits with NEI models resulted in ionisation parameters $\gtrsim 10^{12} \mathrm{~s} \mathrm{~cm}^{-3}$, consistent with a plasma in CIE. For this reason we adopted a pure Fe vnei model with the ionisation parameter fixed at $1 \times 10^{12} \mathrm{~s} \mathrm{~cm}^{-3}$. This yielded an acceptable fit with $\chi_{v}^{2}=1.20$, however, residuals remained in the $0.5-0.7 \mathrm{keV}$ range, suggesting the presence of $O$. Therefore, we added a second pure $\mathrm{O}$ vnei component to the model, with $\tau$ again fixed to $1 \times 10^{12} \mathrm{~s} \mathrm{~cm}^{-3}$, resulting in an improved fit with $\chi_{v}^{2}=1.11$, shown in Fig. 9-right with fit results given in Table 3 . The physical interpretation of the results is discussed in Sect. 5.2.2.
Table 2. Spectral fit results for MCSNR J0506-7025.

\begin{tabular}{|c|c|c|}
\hline Component & Parameter & Value \\
\hline \multicolumn{3}{|c|}{ vpshock (shell) + vnei (Fe core) } \\
\hline $\begin{array}{l}\text { phabs } \\
\text { vphabs }^{b}\end{array}$ & $\begin{array}{l}N_{\mathrm{H}, \mathrm{Gal}}\left(10^{22} \mathrm{~cm}^{-2}\right) \\
N_{\mathrm{H}, \mathrm{LMC}}\left(10^{22} \mathrm{~cm}^{-2}\right)\end{array}$ & $\begin{array}{l}0.08^{a} \\
<0.01\end{array}$ \\
\hline vpshock $^{b}$ & $\begin{array}{l}k T \\
\tau_{u}\left(10^{11} \mathrm{~s} \mathrm{~cm}^{-3}\right) \\
E M\left(10^{57} \mathrm{~cm}^{-3}\right)\end{array}$ & $\begin{array}{r}0.48(0.30-0.74) \\
0.9(0.4-3.0) \\
1.7(0.92-3.65)\end{array}$ \\
\hline \multirow[t]{2}{*}{ vnei } & $\begin{array}{l}k T_{\mathrm{Fe}} \\
\tau\left(10^{12} \mathrm{~s} \mathrm{~cm}^{-3}\right) \\
n_{\mathrm{e}} n_{\mathrm{Fe}} V\left(10^{53} \mathrm{~cm}^{-3}\right)\end{array}$ & $\begin{array}{r}0.78(0.76-0.79) \\
1.0(\text { fixed }) \\
1.15(1.12-1.24)\end{array}$ \\
\hline & $\begin{array}{l}F_{\mathrm{X}, \text { total }}{ }^{c}\left(10^{-13} \mathrm{erg} \mathrm{s}^{-1} \mathrm{~cm}^{-2}\right) \\
L_{\mathrm{X}, \text { shell }^{d}}\left(10^{34} \mathrm{erg} \mathrm{s}^{-1}\right) \\
L_{\mathrm{X}, \mathrm{Fe}}{ }^{d}\left(10^{34} \mathrm{erg} \mathrm{s}^{-1}\right)\end{array}$ & $\begin{array}{l}4.4 \\
8.4 \\
9.5\end{array}$ \\
\hline Fit statistic & $\chi_{v}^{2}$ & 1.16 (472 d.o.f) \\
\hline \multicolumn{3}{|c|}{ vsedov (shell) + vnei (Fe core) } \\
\hline $\begin{array}{l}\text { phabs } \\
\text { vphabs }^{b}\end{array}$ & $\begin{array}{l}N_{\mathrm{H}, \mathrm{Gal}}\left(10^{22} \mathrm{~cm}^{-2}\right) \\
N_{\mathrm{H}, \mathrm{LMC}}\left(10^{22} \mathrm{~cm}^{-2}\right)\end{array}$ & $\begin{array}{l}0.08^{a} \\
<0.07\end{array}$ \\
\hline vsedov $^{b}$ & $\begin{array}{l}k T \\
\tau_{u}\left(10^{11} \mathrm{~s} \mathrm{~cm}^{-3}\right) \\
E M\left(10^{58} \mathrm{~cm}^{-3}\right)\end{array}$ & $\begin{array}{r}0.18(0.15-0.37) \\
4.89(>2.49) \\
1.07(0.28-1.49)\end{array}$ \\
\hline vnei & $\begin{array}{l}k T_{\mathrm{Fe}} \\
\tau\left(10^{12} \mathrm{~s} \mathrm{~cm}^{-3}\right) \\
n_{\mathrm{e}} n_{\mathrm{Fe}} V\left(10^{53} \mathrm{~cm}^{-3}\right)\end{array}$ & $\begin{array}{r}0.80(0.77-0.82) \\
1.0 \text { (fixed) } \\
1.09(0.97-1.20)\end{array}$ \\
\hline & $\begin{array}{l}F_{\mathrm{X}, \text { total }}{ }^{c}\left(10^{-13} \mathrm{erg} \mathrm{s}^{-1} \mathrm{~cm}^{-2}\right) \\
L_{\mathrm{X}, \text { shell }}{ }^{d}\left(10^{35} \mathrm{erg} \mathrm{s}^{-1}\right) \\
L_{\mathrm{X}, \mathrm{Fe}}{ }^{d}\left(10^{34} \mathrm{erg} \mathrm{s}^{-1}\right)\end{array}$ & $\begin{array}{l}4.5 \\
1.8 \\
8.8\end{array}$ \\
\hline Fit statistic & $\chi_{v}^{2}$ & 1.11 (472 d.o.f) \\
\hline
\end{tabular}

Notes. See text for description of the models. The numbers in parentheses are the $90 \%$ confidence intervals. ${ }^{(a)}$ Fixed to the Galactic column density from the Dickey \& Lockman (1990) H I maps. ${ }^{(b)}$ Absorption and thermal component abundances fixed to those of the LMC. ${ }^{(c)} 0.3-10 \mathrm{keV}$ absorbed X-ray flux. ${ }^{(d)} 0.3-10 \mathrm{keV}$ de-absorbed X-ray luminosity, adopting a distance of $50 \mathrm{kpc}$ to the LMC.

\section{Results}

\subsection{MCSNR J0506-7025}

\subsubsection{Multi-wavelength morphology}

The X-ray emission from MCSNR J0506-7025 is characterised by a bright core in the $0.7-1.1 \mathrm{keV}$ range, surrounded by a soft shell evident in the $0.3-0.7 \mathrm{keV}$ band (Fig. 1-top left). The $\mathrm{X}$-ray spectral analysis has shown that the enhanced $0.7-1.1 \mathrm{keV}$ emission in the core is due to an Fe rich gas, most likely reverse shock heated Fe ejecta. Similarly, the soft shell is consistent with swept-up and shock-heated ISM, typical of an evolved SNR. This Fe core/soft shell morphology has been observed in several other evolved LMC SNRs, including DEM L238 and DEM L249 (Borkowski et al. 2006) and MCSNR J0508-6902 (Bozzetto et al. 2014b). The 0.7-1.1 keV emission is not confined to a clear, well-defined core, with emission spreading in both northernly and southernly directions. This may be due to ejecta from the $\mathrm{Fe}$ core being smeared out across the remnant 

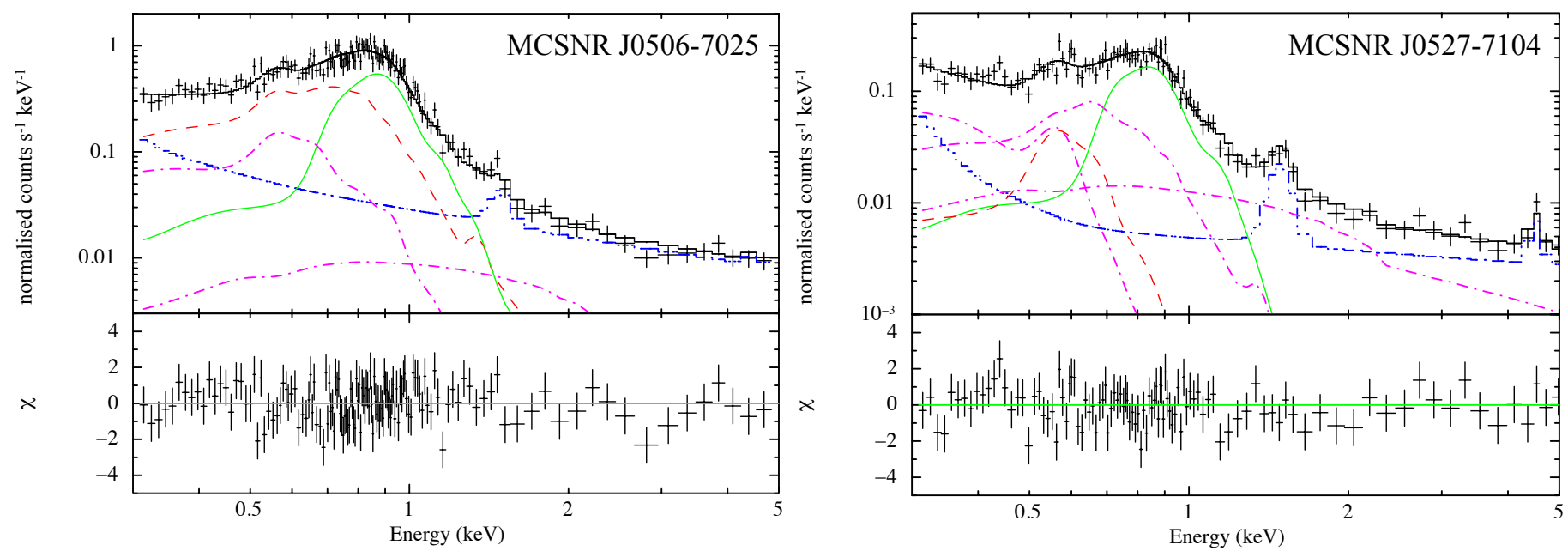

Fig. 9. Left: best-fit vsedov+vnei model to the X-ray spectrum of MCSNR J0506-7025. The green solid line represents the pure Fe component, the dashed red line shows shell component, the magenta dash-dot lines indicate the AXB components, and the blue dash-dot-dot-dot line shows the combined contributions of the QPB, instrumental fluorescence lines, and electronic noise. Right: best-fit vnei+vnei model to the X-ray spectrum of MCSNR J0527-7104. Same as left except the dashed red line represents the pure O component. In both cases, only the EPIC-pn spectrum is shown for clarity. Best-fit parameters for MCSNR J0506-7025 and MCSNR J0527-7104 are given in Tables 2 and 3, respectively.

Table 3. Spectral fit results for MCSNR J0527-7104.

\begin{tabular}{|c|c|c|}
\hline Component & Parameter & Value \\
\hline \multicolumn{3}{|c|}{ vnei + vnei } \\
\hline \multirow{2}{*}{$\begin{array}{l}\text { phabs } \\
\text { vphabs }^{b}\end{array}$} & $N_{\mathrm{H}, \mathrm{Gal}}\left(10^{22} \mathrm{~cm}^{-2}\right)$ & $0.07^{a}$ \\
\hline & $N_{\mathrm{H}, \mathrm{LMC}}\left(10^{22} \mathrm{~cm}^{-2}\right)$ & $<0.05$ \\
\hline \multirow[t]{3}{*}{ vnei $(F e)$} & $k T_{\mathrm{Fe}}$ & $0.71(0.68-0.73)$ \\
\hline & $\tau\left(10^{12} \mathrm{~s} \mathrm{~cm}^{-3}\right)$ & 1.0 (fixed) \\
\hline & $n_{\mathrm{e}} n_{\mathrm{Fe}} V\left(10^{52} \mathrm{~cm}^{-3}\right)$ & $2.7(2.5-2.9)$ \\
\hline \multirow[t]{6}{*}{ vnei $(O)$} & $k T_{\mathrm{O}}$ & $0.18(0.14-0.23)$ \\
\hline & $\tau\left(10^{12} \mathrm{~s} \mathrm{~cm}^{-3}\right)$ & 1.0 (fixed) \\
\hline & $n_{\mathrm{e}} n_{\mathrm{O}} V\left(10^{53} \mathrm{~cm}^{-3}\right)$ & $2.2(1.6-3.8)$ \\
\hline & $F_{\mathrm{X}}^{c}\left(10^{-14} \mathrm{erg} \mathrm{s}^{-1} \mathrm{~cm}^{-2}\right)$ & 7.3 \\
\hline & $L_{\mathrm{X}, \mathrm{Fe}}{ }^{d}\left(10^{34} \mathrm{erg} \mathrm{s}^{-1}\right)$ & 2.2 \\
\hline & $L_{\mathrm{X}, \mathrm{O}}{ }^{d}\left(10^{34} \mathrm{erg} \mathrm{s}^{-1}\right)$ & 0.5 \\
\hline Fit statistic & $\chi_{v}^{2}$ & 1.11 (475 d.o.f.) \\
\hline
\end{tabular}

Notes. See text for description of the model. The numbers in parentheses are the $90 \%$ confidence intervals. ${ }^{(a)}$ Fixed to the Galactic column density from the Dickey \& Lockman (1990) H I maps. ${ }^{(b)}$ Absorption component abundances fixed to those of the LMC. ${ }^{(c)} 0.3-10 \mathrm{keV}$ absorbed X-ray flux. ${ }^{(d)} 0.3-10 \mathrm{keV}$ de-absorbed X-ray luminosity, adopting a distance of $50 \mathrm{kpc}$ to the LMC.

or simply indicate an enhancement of the shell emission. While the northern $0.7-1.1 \mathrm{keV}$ emission is largely correlated with the softer shell emission, the southern extension is not, suggesting that the Fe gas is not confined to a well-defined core.

The shell emission is elliptical, more extended in the northsouth direction than east-west. There are some brightness enhancements, notably in a knot at the very east and along the western front. These regions are correlated with enhanced radio emission (Fig. 3), and regions of highest polarisation (Fig. 8). Well-defined shell-like [S II] enhancements (see Fig. 1-top right) corresponding to regions where $[\mathrm{S} \mathrm{II}] / \mathrm{H} \alpha>0.67$, are detected ahead of the correlated X-ray and radio emission along the western front. These morphologies are consistent with the blast wave

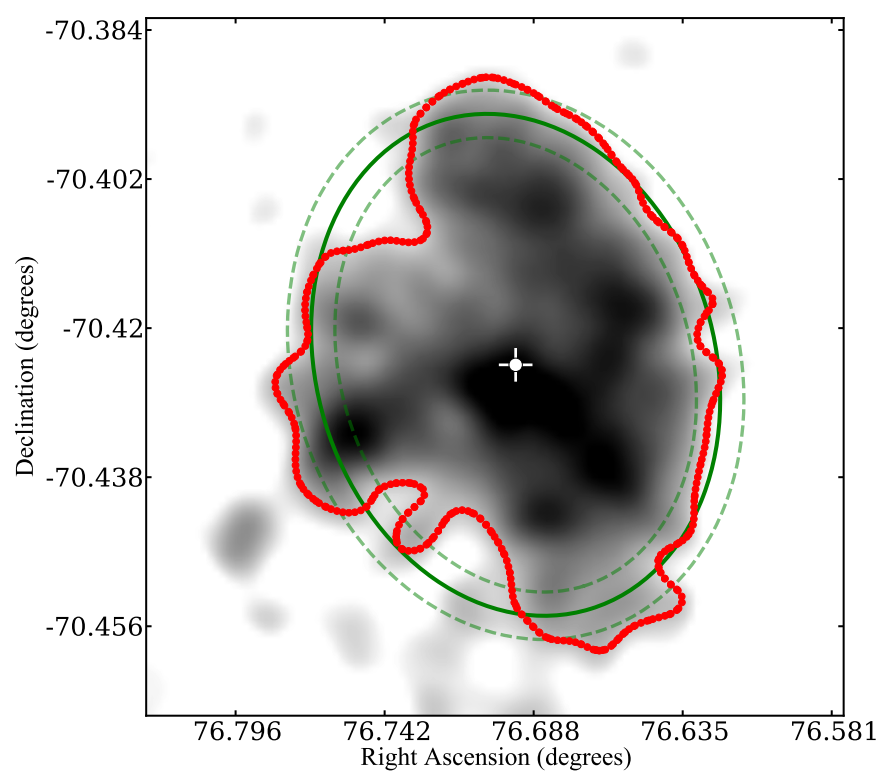

Fig. 10. Combined 0.3-0.7 keV EPIC image of MCSNR J0506-7025. The red points delineate the contour level corresponding to $3 \sigma$ above the average background surface brightness. The green solid line shows the best-fit ellipse to the contour, with the dashed lines indicating the $1 \sigma$ error on the fit. The white plus-sign indicates the best-fit centre of the SNR.

having encountered some denser ambient medium in these regions. To investigate this we made use of data from the SAGE survey of the LMC (see Sect. 2.4.1). The $24 \mu \mathrm{m}$ image of MCSNR J0506-7025 is shown in Fig. 1-bottom right, and reveals enhanced dust emission ahead of the eastern, western, and southern fronts. We investigated this further using data from the H I survey of the LMC (see Sect. 2.4.2). We extracted channel maps centred on MCSNR J0506-7025, shown in Fig. 11. The SNR is located at the GS $30 \mathrm{HI}$ shell (Kim et al. 1999), with distribution of $\mathrm{HI}$ in the $226 \mathrm{~km} \mathrm{~s}^{-1}, 231 \mathrm{~km} \mathrm{~s}^{-1}, 236 \mathrm{~km} \mathrm{~s}^{-1}$ channel maps consistent with the expectation of ambient density enhancements to the east and west of MCSNR J0506-7025. Along the western front, the magnetic field vectors are oriented 

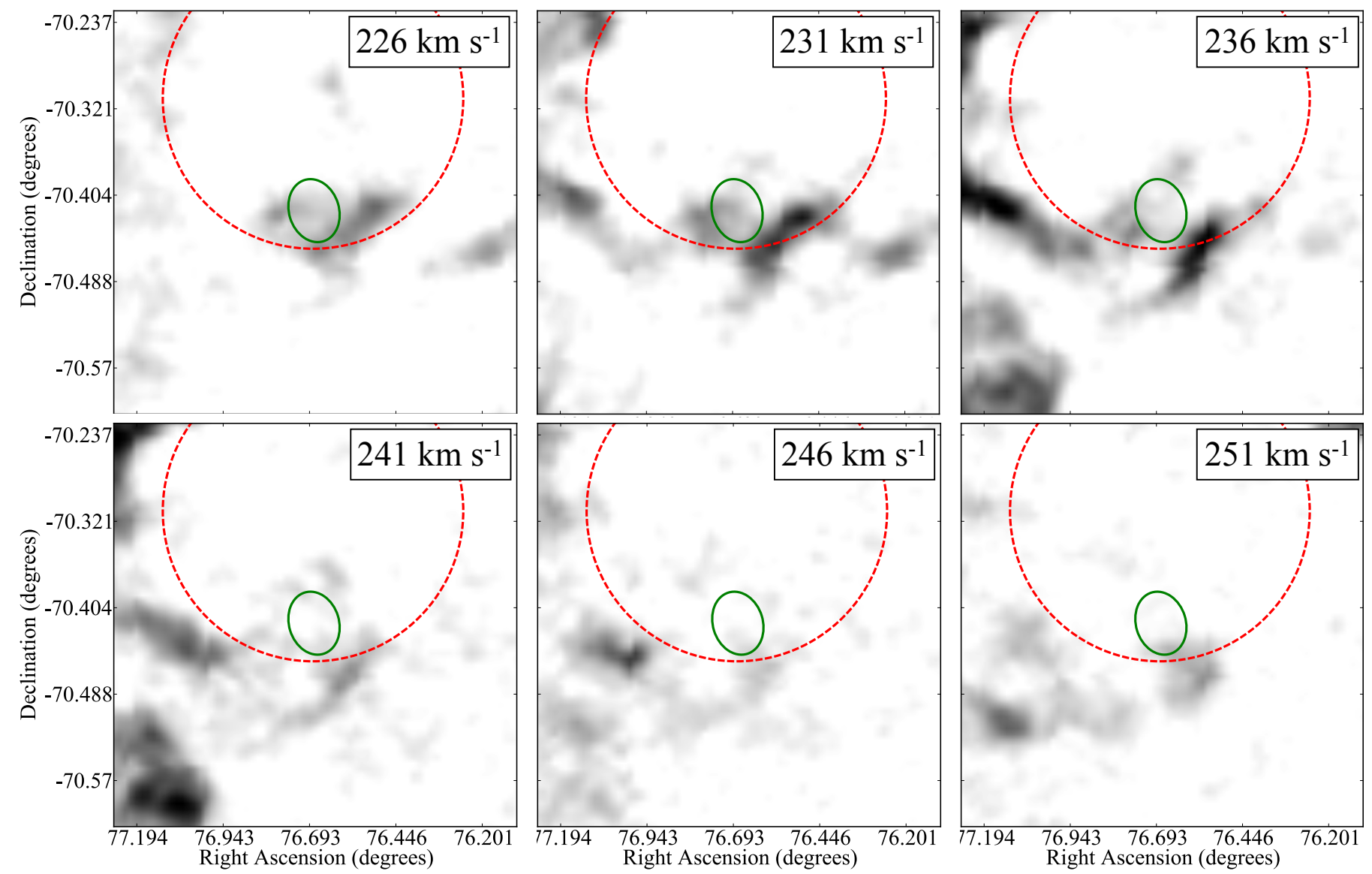

Fig. 11. H I channel maps of the MCSNR J0506-7025 region. The dimensions of the SNR are shown by the green ellipse in each panel. The red dashed line indicates the approximate position of the giant H I shell GS 30 (Kim et al. 1999). The heliocentric velocities are also given.

tangential to the shock front, typical of older remnants where there is compression of the interstellar ambient field. The field vectors in the knot to the east show a radial orientation. We also note that faint radio emission was found to extend $\sim 1^{\prime}-2^{\prime}$ beyond the observed X-ray shell to the south (see Figs. 3 and 8). It is unclear if this is associated with MCSNR J0506-7025 or is unrelated background emission, possibly due to the nearby H II region. The radio spectral index map shown in Fig. 4-left indicates a flatter spectrum in this feature, more consistent with a thermal origin. For this reason, and given the absence of correlated X-ray and characteristic optical emission, it is likely that the extended radio feature is unrelated to the SNR.

To estimate the size of the X-ray remnant we determined the average background surface brightness and corresponding standard deviation $(\sigma)$ in the $0.3-0.7 \mathrm{keV}$ band. We then defined the edge of the SNR as regions where the extended emission surface brightness rises to $3 \sigma$ above the average background and fitted an ellipse to this contour. The error on the fit was determined by quantifying the standard deviation of points on the contour from the best-fit ellipse. We determined a best-fit ellipse centred on the $\mathrm{J} 2000$ coordinates RA $=05^{\mathrm{h}} 06^{\mathrm{m}} 47.30^{\mathrm{s}}$ and Dec $=-70^{\mathrm{d}} 25^{\mathrm{m}} 29.7^{\mathrm{s}}$, of size $2{ }^{\prime} .89( \pm 0.35) \times 3{ }^{\prime} 71( \pm 0.35)$, corresponding to $42.1( \pm 5.0) \mathrm{pc} \times 54.0( \pm 5.0) \mathrm{pc}$ at the LMC distance, with the major axis rotated $\sim 17^{\circ}$ east of north. The best-fit dimensions and error are shown in Fig. 10.

\subsubsection{X-ray emission}

The X-ray spectrum of MCSNR J0506-7025 was found to be described well by either the vpshock+vnei or vsedov+vnei model with marginal difference in the fit-statisitic $\left(\chi_{v}^{2}=1.16\right.$ and $\chi_{v}^{2}=1.11$, respectively), as shown in Table 2 . In each case, the best-fit absorption due to foreground material in the LMC was negligible, with only upper limits determined. As argued in Maggi et al. (2016), the negligible foreground absorption can be interpreted as the SNR being located on the near side of the LMC. Therefore, it is possible that the apparent correlation of MCSNR J0506-7025 with GS 30, shown in Fig. 11, is simply a chance alignment. Moreover, we argue in the forthcoming sections for a Type Ia origin for MCSNR J0506-7025 and, therefore, the association of the remnant with an $\mathrm{H}$ I giant shell is not expected. However, given the correlation between the bright regions of X-ray material with denser material in the H I maps (see Sect. 5.1.1, it is likely that there is at least some interaction of the remnant and GS 30.

We now discuss the physical interpretation of the shell and ejecta emission components.

\section{Shell emission}

The best-fit plasma temperatures for the shell components of each model agree within the $90 \%$ confidence intervals, and are consistent with other evolved SNRs in the LMC (see Maggi et al. 2014; Kavanagh et al. 2015, for example). The shell ionisation parameters inferred from the fits are also compatible, though only a lower limit could be determined for the vsedov component.

Using our fit results we can estimate physical parameters of MCSNR J0506-7025 using the Sedov dynamical model (see Sasaki et al. 2004; Kavanagh et al. 2015, for examples). 
Table 4. Physical properties of MCSNR J0506-7025 derived from the Sedov model.

\begin{tabular}{ccccc}
\hline \hline $\begin{array}{c}n_{0} \\
\left(10^{-2} \mathrm{~cm}^{-3}\right)\end{array}$ & $\begin{array}{c}v_{\mathrm{s}} \\
\mathrm{km} \mathrm{s}^{-1}\end{array}$ & $\begin{array}{c}t \\
(\mathrm{kyr})\end{array}$ & $\begin{array}{c}M \\
\left(M_{\odot}\right)\end{array}$ & $\begin{array}{c}E_{0} \\
\left(10^{51} \mathrm{erg}\right)\end{array}$ \\
\hline $2.6-7.0$ & $354-557$ & $16-28$ & $46-155$ & $0.07-0.84$ \\
\hline
\end{tabular}

The X-ray shell of the remnant is delineated by an ellipse (see Sect. 5.1.1), with semi-major and semi-minor axes of $27.0 \mathrm{pc}$ and $21.0 \mathrm{pc}$, respectively. Assuming these are the first and second semi-principal axes of an ellipsoid describing MCSNR J0506-7025, and that the third semi-principal axis is in the range $27.0-21.0 \mathrm{pc}$, we determined the volume $(V)$ limits for the remnant and their corresponding effective radii $\left(R_{\text {eff }}\right)$ to be $(1.5-1.9) \times 10^{60} \mathrm{~cm}^{3}$ and $22.8-24.8 \mathrm{pc}$, respectively.

The best fit shell X-ray temperature (see Table 2) corresponds to a shock velocity

$v_{\mathrm{s}}=\left(\frac{16 k T_{\mathrm{s}}}{3 \mu}\right)^{0.5}$

where $k T_{\mathrm{s}}$ is the postshock temperature and $\mu$ is the mean mass per free particle. For a fully ionised plasma with LMC abundances, $\mu=0.61 m_{\mathrm{p}}$, resulting in a shock velocity of $v_{\mathrm{s}}=$ 388 (354-557) $\mathrm{km} \mathrm{s}^{-1}$. The age of the remnant can now be determined from the self-similarity solution:

$v_{s}=\frac{2 R}{5 t}$,

where $R=R_{\text {eff }}$ and $t$ is the age of the remnant. This gives an age range of 16-28 kyr. The pre-shock $\mathrm{H}$ density $\left(n_{\mathrm{H}, 0}\right)$ in front of the blast wave can be determined from the emission measure (EM, see Table 2). Evaluating the emission integral over the Sedov solution using the approximation for the radial density distribution of Kahn (1975) gives

$\int n_{\mathrm{e}} n_{\mathrm{H}} \mathrm{d} V=E M=2.07\left(\frac{n_{\mathrm{e}}}{n_{\mathrm{H}}}\right) n_{\mathrm{H}, 0}^{2} V$,

where $n_{\mathrm{e}}$ and $n_{\mathrm{H}}$ are electron and hydrogen densities, respectively, $V$ is the volume (e.g., Hamilton et al. 1983). Taking $n_{\mathrm{e}} / n_{\mathrm{H}}=1.21$ and the determined range of volumes, this equation yields $n_{\mathrm{H}, 0}=(2.4-6.4) \times 10^{-2} \mathrm{~cm}^{-3}$. Since the pre-shock density of nuclei is given as $n_{0} \sim 1.1 n_{\mathrm{H}, 0}$, it follows that $n_{0}=$ $(2.6-7.0) \times 10^{-2} \mathrm{~cm}^{-3}$. Hence, the SNR is expanding into a fairly rarified environment. The initial explosion kinetic energy $\left(E_{0}\right)$ can be determined from the equation:

$R=\left(\frac{2.02 E_{0} t^{2}}{\mu_{n} n_{0}}\right)^{1 / 5}$

where $\mu_{n}$ is the mean mass per nucleus $\left(=1.4 m_{\mathrm{p}}\right)$. This results in an initial explosion energy in the range $(0.07-0.84) \times 10^{51} \mathrm{erg}$. This is lower than the canonical $10^{51}$ erg but consistent with several Type Ia SNRs in the LMC (e.g., Hendrick et al. 2003; Bozzetto et al. 2014b). The swept-up mass contained in the shell is given simply by $M=V \mu_{n} n_{0}$, which is evaluated to (46-155) $M_{\odot}$. This large amount of swept-up material supports our original assumption that MCSNR J0506-7025 is into the Sedov phase of its evolution. All the derived parameters of MCSNR J0506-7025 are summarised in Table 4.

Using the determined values of $t$ and $n_{0}$ we estimated the expected value of the ionisation parameter $(\tau)$ in the vsedov component of the spectral fit. Given that $\tau$ is the product of the electron density immediately behind the shock front and the remnant age (Borkowski et al. 2001), it can be written $\tau=n_{\mathrm{e}} t \approx 4.8 n_{\mathrm{H}, 0} t$. We determined the expected $\tau$ to be in the range $(0.60-2.52) \times 10^{11} \mathrm{~cm}^{-3} \mathrm{~s}$, which is in agreement with the $\tau$ determined in the spectral fits (see Table 2), though the fitted ionisation parameter is poorly constrained.

\section{Ejecta emission}

The interior emission of MCSNR J0506-7025 was modelled with a thermal plasma model consisting of pure Fe. We found that a CIE plasma with best-fit of $k T_{\mathrm{Fe}}=0.78(0.76-0.79) \mathrm{keV}$ in the vpshock+vnei model and $k T_{\mathrm{Fe}}=0.80(0.77-0.82) \mathrm{keV}$ in the vsedov+vnei model provided a good fit to the spectrum (see Table 2). The plasma temperatures and emission measures for both models are in agreement within the $90 \%$ confidence intervals. We proceed adopting the $\mathrm{Fe}$ emission parameters of the vsedov+vnei model. The mass of $\mathrm{Fe}$ in the core can be determined from the emission measure of the gas and the volume. This method is described in detail in Kosenko et al. (2010), Bozzetto et al. (2014b), Maggi et al. (2014), with the Fe mass given by the equation

$M_{\mathrm{Fe}}=\left(V_{\mathrm{Fe}} E M_{\mathrm{Fe}}\right)^{0.5}\left(n_{\mathrm{e}} / n_{\mathrm{Fe}}\right)^{-0.5} m_{\mathrm{U}} A_{\mathrm{Fe}}$,

where $V_{\mathrm{Fe}}$ is the volume occupied by the Fe, $E M_{\mathrm{Fe}}=n_{\mathrm{e}} n_{\mathrm{Fe}} V$ is the emission measure of the Fe gas, $n_{\mathrm{e}} / n_{\mathrm{Fe}}$ is the electron to Feion ratio, $m_{\mathrm{U}}$ is the atomic mass unit, and $A_{\mathrm{Fe}}$ is the atomic mass of Fe. $E M_{\mathrm{Fe}}$ is obtained from the normalisation of the Fe component and is listed in Table 2 . The $n_{\mathrm{Fe}} / n_{\mathrm{H}}$ value is calculated from the Fe abundance parameter of the model.

The determination of $V_{\mathrm{Fe}}$ presents a problem. As discussed in Sect. 5.1.1, the emission in the $0.7-1.1 \mathrm{keV}$ range is not confined to a central well-defined core, with extensions evident to the north and south. It is unclear if these extensions are due to the Fe-rich ejecta or to enhancements in the shell emission. Ideally, we could perform a spatially resolved spectral analysis from various regions around the remnant to determine where an Fe L-shell bump is present. Unfortunately, because of the count statistics, this is not possible with the current data set. However, we can get an estimate of the distribution of Fe by comparing count rates $(N)$ in specific energy bands across the remnant and creating an X-ray colour map. To do this we made use of the Weighted Voronoi Tessellation (WVT) binning algorithm by Diehl \& Statler (2006) ${ }^{11}$, which is a generalisation of the Cappellari \& Copin (2003) Voronoi binning algorithm. This is an adaptive image binning technique that uses weighted Voronoi tessellations to bin the image to a user defined signalto-noise $(\mathrm{S} / \mathrm{N})$ per bin while keeping the bin sizes as compact as possible. The WVT binning tools provide an X-ray colour map facility which compares the counts in two energy bands to gain a picture of the variation in X-ray colour over the remnant. We defined the two energy bands, a soft band $S=0.3-0.7 \mathrm{keV}$ (where the shell dominates) and a hard band $H=0.7-1.0 \mathrm{keV}$ (where the Fe L-shell bump peaks), and the colour $S / H$ is then given by $N_{0.3-0.7 \mathrm{keV}} / N_{0.7-1.0 \mathrm{keV}}$. We set a target $\mathrm{S} / \mathrm{N}$ of 5 so that the bins give a useful estimate of $S / H$ in each bin while producing small enough bins to get a picture of the Fe distribution. The resulting WVT X-ray colour map is shown in Fig. 12. It is already clear from the image that $\mathrm{Fe}$ dominates the core, as expected. However, we must decide where to apply the cut

\footnotetext{
11 See also http://WwW.phy.ohiou.edu/ diehl/WVT/index. html\#home.
} 


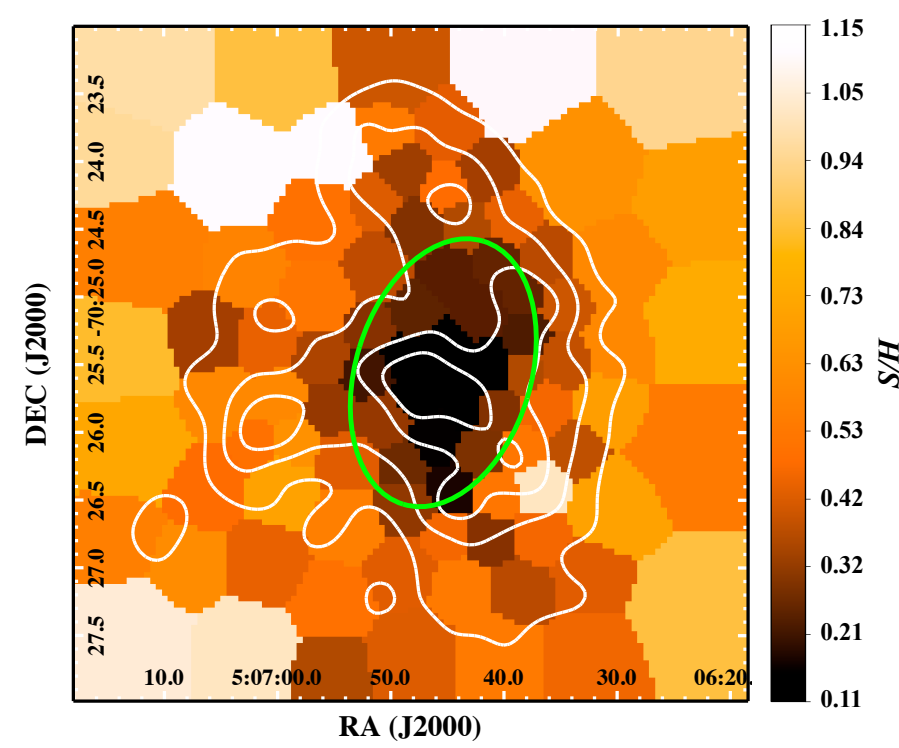

Fig. 12. WVT X-ray colour map of MCSNR J0506-7025. The tessellates are defined so that each has a $\mathrm{S} / \mathrm{N}$ of 5 . Lower values of $S / H$ indicate higher contributions from Fe L-shell emission. The white contours show the $0.3-0.7 \mathrm{keV}$ counters from Fig. 1-top right and the green ellipse outlines the Fe L-shell emission regions, set to $S / H<0.3$ (see text).

to the Fe dominating regions. To do this we performed spectral simulations assuming that the best-fit core and shell component parameters are constant over the remnant (see Table 2), allowing only the relative normalisation between the shell and core components to vary. These simulations suggested that the pure Fe component becomes significant when $S / H$ drops below $\sim 0.35$. Given the relatively low $\mathrm{S} / \mathrm{N}$ per bin, we set a conservative upper limit at $S / H<0.3$. The majority of tessellates with $S / H<0.3$ are aggregated in the centre of the SNR. We traced an ellipse around these tessellates by eye, and assume that this represents the projected volume of the Fe ejecta (green ellipse in Fig. 12). The resulting dimensions of the central region are $\sim 19.0 \mathrm{pc} \times 29.6 \mathrm{pc}$. We assume an ellipsoidal morphology with a third semi-axis equal to the mean of the semi-major and semiminor axes $(12.2 \mathrm{pc})$. If the actual morphology is oblate or prolate, the volume would be $\sim 22 \%$ higher or lower, respectively.

As discussed in Hughes et al. (2003), there are two limiting cases for the value of the $n_{\mathrm{e}} / n_{\mathrm{Fe}}$ ratio, depending on the level of admixture of $\mathrm{H}$ in the ejecta. If there is no $\mathrm{H}$ in the ejecta, then the number of free electrons per Fe ion only depends on the average ionisation state of the Fe. For a plasma in CIE at $k T=0.80 \mathrm{keV}(\log T \sim 6.95)$ the average ionisation is 18.3 for $\mathrm{Fe}$ (Shull \& van Steenberg 1982). Then, from Eq. (6, the Fe mass is in the range 1.6-1.7 $M_{\odot}$. Alternatively, one can assume that a similar mass of $\mathrm{H}$ is mixed into the $\mathrm{Fe}$ rich ejecta. Therefore, the number density of Fe over $\mathrm{H}$ is $1 / 56$, and the average number of electrons per Fe ion is 74.3. It follows from Eq. (6) that the Fe mass is in the range 0.8-0.9 $M_{\odot}$ if there is a comparable mass of $\mathrm{H}$ to $\mathrm{Fe}$ in the ejecta. However, the ejecta may not occupy the entire volume of the projected morphology which would be expected if the ejecta were clumpy. In this case, the emitting volume must be modified by a filling factor of $\sim 0.4$ (see Kosenko et al. 2010, and references therein), in which case the $\mathrm{Fe}$ content reduces by a factor of $\sqrt{0.4} \approx 0.63$ giving $1.0-1.1 M_{\odot}$ and $0.5-0.6 M_{\odot}$ for the pure Fe and $\mathrm{Fe}+\mathrm{H}$ mixture cases, respectively. For a Type Ia explosion, we would expect that about half of the ejecta mass is Fe, $\sim 0.7 M_{\odot}$ (e.g., Iwamoto et al. 1999). Therefore, our results point to a the clumpy ejecta of a Type Ia explosion with some admixture of $\mathrm{H}$ in the Fe-rich ejecta.

\subsection{MCSNR J0527-7104}

\subsubsection{Multi-wavelength morphology}

The extent of MCSNR J0527-7104 is best observed in the optical regime, with a clear elliptical shell with $[\mathrm{S} \mathrm{II}] / \mathrm{H} \alpha$ consistent with shock excitation. However, as reported in KSP13, the projected elliptical shape is incomplete at the northwestern end with only very faint filamentary structure visible. Comparison of the optical shell to dust emission in the region suggests that MCSNR J0527-7104 has cleared out the dust, with the shell edge corresponding very closely to a dust cavity (Fig. 2-bottom right).

The X-ray morphology of MCSNR J0527-7104 is extremely unusual. The emission extends from the southeastern optical shell, right through the southeast-northwest axis of symmetry, appearing to escape or blow-out the northwestern shell (Fig. 2-bottom left). Blow-outs have been observed at various wavelengths in other LMC remnants, namely N 11L and N 86 (Murphy Williams et al. 1999; Crawford et al. 2008). We obtained the MIPS $24 \mu \mathrm{m}$ image of MCSNR J0527-7104 (see Sect. 2.4.1), shown in Fig. 2-bottom right, which indicates a possible reason for the blow-out in the northwest. The dust emission is noticeably absent outside the northwestern shell which might indicate a significantly lower ISM density in the northwest as compared to other regions of the SNR. We investigate this further using data from the H I survey of the LMC (see Sect. 2.4.2) by extracting channel maps centred on MCSNR J0527-7104, shown in Fig. 13. The distribution of $\mathrm{HI}$ in the $226 \mathrm{~km} \mathrm{~s}^{-1}$, $231 \mathrm{~km} \mathrm{~s}^{-1}, 236 \mathrm{~km} \mathrm{~s}^{-1}$, and $241 \mathrm{~km} \mathrm{~s}^{-1}$ panels indicates an $\mathrm{H} \mathrm{I}$ cavity immediately to the northwest of the SNR. A similar explanation has been proposed for the blow-out from N 11L (Murphy Williams et al. 1999). For this reason, we suggest that the blast wave of MCSNR J0527-7104 has blown-out into this low density cavity, allowing the shocked ejecta gas to escape behind it. Given the low density conditions in the ejecta, and that the $\mathrm{Fe}$ rich gas can only lose energy via inefficient radiative cooling, the ejecta can remain X-ray bright as the gas escapes the core into the cavity.

The absence of notable soft X-ray emission within the optical shell with abundances consistent with swept-up ISM could point to either a high absorbing column in the direction of MCSNR J0527-7104 or that the remnant is close to the transition into the radiative phase and the shell has become either too cool to emit X-rays or too faint to be detectable. The low value of $N_{\mathrm{H}, \mathrm{LMC}}$ in the spectral fits (see Table 3) suggests the latter. Moreover, the prominent [S II] and significant [O III] indicates that at least part of the shell is radiative, and some fraction of the gas has cooled down below X-ray emitting temperatures. Other LMC remnants to exhibit similar properties include MCSNR J0508-6830 and MCSNR J0511-6759 (Maggi et al. 2014).

Other members of the evolved Fe-rich SNR class in the LMC also exhibit irregular/elongated cores, though somewhat less pronounced than that of MCSNR J0527-7104. We have argued that the unusual core morphology of MCSNR J0527-7104 is the result of a blowout with ejecta escaping into a low density cavity. In other members of the sample, there is no compelling evidence that the irregular/elongated cores resulted from a blowout since the ejecta always appear internal to the optical/X-ray shells. 

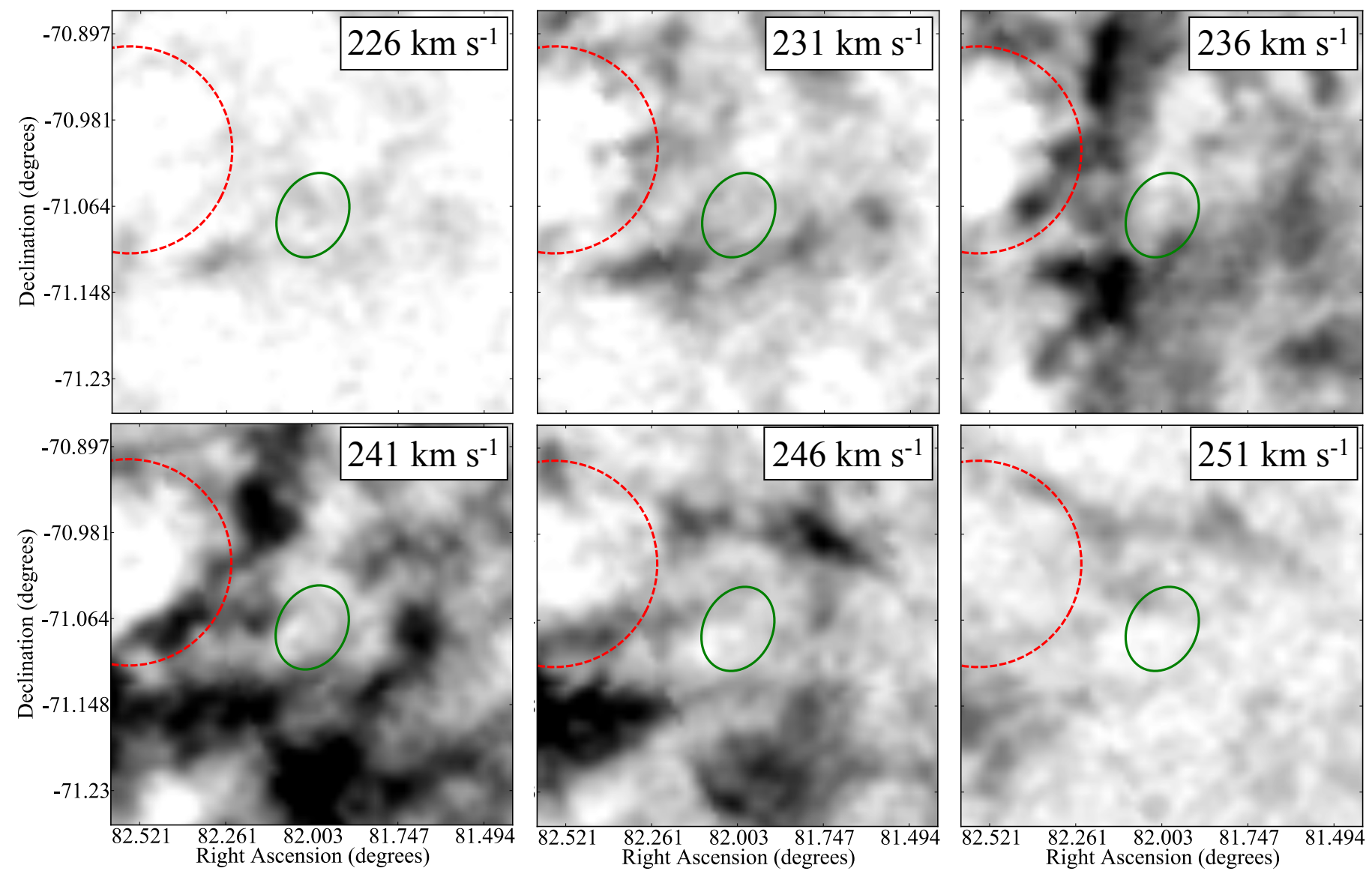

Fig. 13. H I channel maps of the MCSNR J0527-7104 region. The dimensions of the SNR determined in KSP13 are shown by the green ellipse in each panel. The red dashed line indicates the approximate position of the giant H I shell GS 70 (Kim et al. 1999) associated with the N 206 star forming region. The heliocentric velocities are also given. In the $226 \mathrm{~km} \mathrm{~s}^{-1}, 231 \mathrm{~km} \mathrm{~s}^{-1}, 236 \mathrm{~km} \mathrm{~s}^{-1}$, and $241 \mathrm{~km} \mathrm{~s}^{-1}$ panels there is a noticeable reduction in $\mathrm{H}$ I density to the northwest of the shell.

Remnants such as MCSNR J0506-7025, discussed above, and MCSNR J0508-6902 (Bozzetto et al. 2014b) display elongated Fe-rich cores, approximately aligned with the elongated axis of symmetry of the shells, suggesting a common origin. The core of MCSNR J0527-7104 is also elongated along the shell's axis of symmetry and, though the dimensions of the core were likely increased by the blowout, the ellipsoidal shell suggests that some initial elongation could have also been present. Possible external influences which could cause the elongation are ambient density inhomogeneities or ISM magnetic field orientation. Explosion asymmetries offer an intrinsic explanation for the elongation, with Type Ia explosions thought to be sparked off-set from centre (e.g., Maeda 2012, and references therein). Explosion asymmetries could also explain the Fe-ejecta "schrapnel" observed in MCSNR J0511-6759 outside the bulk Fe core but behind the eastern shell front (Maggi et al. 2014). A truly rigorous treatment of the distribution in the Fe cores of these objects requires deep observations by Chandra which offers superior subarcsecond resolution. Unfortunately, those remnants with irregular/elongated cores have yet to be observed by Chandra with sufficient depth to facilitate such an analysis.

\subsubsection{X-ray emission}

\section{Ejecta emission}

The X-ray emission of MCSNR J0527-7104 was found to be entirely ejecta emission with two thermal components representing pure $\mathrm{Fe}$ and pure $\mathrm{O}$ providing a good fit to the $\mathrm{X}$-ray spectrum. We found that the emission was dominated by the Fe component with $k T_{\mathrm{Fe}}=0.71(0.69-0.73) \mathrm{keV}$, with a small contribution from $O$. The temperature of the $\mathrm{O}$ component was found to be lower than that of the $\mathrm{Fe}$ gas, $k T_{\mathrm{O}}=0.17(0.13-0.22) \mathrm{keV}$, but consistent with the peak emissivity temperature of the strongest $\mathrm{O}$ lines in the $0.3-1 \mathrm{keV}$ range (i.e., $k T=0.17 \mathrm{keV}$ ). Given this temperature difference, it is unclear whether the $\mathrm{Fe}$ and $\mathrm{O}$ components are co-spatial. From the SN nucleosynthesis yields of Iwamoto et al. (1999), the O/Fe ratio is expected to be $0.3-0.7$ by number for Type Ia events and $\sim 70$ for CC events. Using the determined emission measures of $\mathrm{O}$ and $\mathrm{Fe}$, and assuming that they are co-spatial results in an $\mathrm{O} / \mathrm{Fe}$ ratio of $\sim 6-15$, closer to that expected for a Type Ia explosion but still incompatible with both scenarios. If the $\mathrm{O}$ and $\mathrm{Fe}$ are not co-spatial then this ratio is not representative of the ejecta. For the purposes of the forthcoming calculations, we assume for simplicity that the $\mathrm{Fe}$ and $\mathrm{O}$ components are not co-spatial and that Fe dominates the interior volume.

We follow the same procedure outlined in Sect. 5.1.2 for the calculation of the ejecta $\mathrm{Fe}$ mass. We fitted an ellipse to the $0.7-1.1 \mathrm{keV}$ extent of the SNR and determined the dimensions of the ejecta emission to be $\sim 1$ ' $1 \times 3$ ' 0 , corresponding to $30.4 \mathrm{pc} \times 86.6 \mathrm{pc}$ at the LMC distance. Given the highly elongated morphology of the ejecta emission, it is far more likely that the depth of the emission is closer to $30.4 \mathrm{pc}$ than $86.6 \mathrm{pc}$. Therefore, we assume that the third dimension of the ellipsoid is $30.4 \mathrm{pc}$, yielding a total ejecta volume $V$ of $1.2 \times 10^{60} \mathrm{~cm}^{3}$.

For our spectral fit results and estimated $V$, a pure heavy element ejecta results in an Fe mass of 1.9-2.1 $M_{\odot}$, about three 
times the Fe mass expected for a Type Ia yield. In the case that an equivalent mass of $\mathrm{H}$ is mixed into the Fe-rich ejecta, the estimated $\mathrm{Fe}$ mass is in the range $0.9-1.0 M_{\odot}$, slightly higher than expected for a Type Ia explosion. Again, it is likely that the ejecta are clumpy and the determined masses must be corrected accordingly (see Sect. 5.1.2), resulting in an estimated $\mathrm{Fe}$ mass of 1.2-1.3 $M_{\odot}$ and 0.6-0.7 $M_{\odot}$ for the pure $\mathrm{Fe}$ and $\mathrm{Fe}+\mathrm{H}$ mixture cases, respectively. It is clear from these results that, a clumpy ejecta with an admixture of $\mathrm{H}$ of similar mass to the $\mathrm{Fe}$ ejecta is required for agreement between our determined Fe mass and that expected from Type Ia explosive nucleosynthesis yields (e.g., Iwamoto et al. 1999).

\section{Summary}

We presented the analysis of new XMM-Newton observations of MCSNR J0506-7025 and MCSNR J0527-7104, and supplemented these with data from the Australian Telescope Compact Array, the Magellanic Cloud Emission Line Survey, Spitzer, and the H I survey of the LMC. The main findings for each remnant can be summarised as follows:

\section{MCSNR J0506-7025}

- We estimated the size of the remnant to be $54.0( \pm 5.0) \mathrm{pc} \times$ $42.1( \pm 5.0) \mathrm{pc}$, with the major axis rotated $\sim 17^{\circ}$ east of north. The shell X-ray emission is highly correlated with the enhanced [S II] and radio emission with a common knot in the east and extended emission along the western front. We found that this is most likely due to the SNR encountering a denser medium in these regions.

- We measured the radio spectral index of MCSNR J0506-7025 using integrated flux measurements, a spectral index map, and T-T plots. Although some variation in the determined spectral index was found depending on the method, all the results point to the emission being non-thermal in nature. The most reliable results, the T-T plot method, showed a spectral index consistent with the standard -0.5 for SNRs.

- Radio polarisation at $6 \mathrm{~cm}$ indicate a higher degree of polarisation along the western front and at the eastern knot, with a mean fractional polarisation across the remnant of $P \cong(20 \pm 6) \%$. The field vectors were oriented tangential to the shock front in the west, typical of older remnants where there is compression of the interstellar ambient field. The field vectors in the knot to the east show a somewhat radial orientation.

- We detected a two-component X-ray emission from MCSNR J0506-7025 with a central core dominated by Fe L-shell emission surrounded by a softer shell. The soft shell is typical of swept-up and shock-heated ISM, consistent with an SNR in the Sedov phase, whereas the Fe component is most likely reverse shock-heated ejecta.

- Using the spectral fit results for the shell and the Sedov self-similar solution, we estimate the age of MCSNR J0506-7025 to be $\sim 16-28 \mathrm{kyr}$, with an initial explosion energy of $(0.07-0.84) \times 10^{51} \mathrm{erg}$. We calculated the mass of Fe contained in the Fe-rich core, which is consistent with expected yields from Type Ia explosive nucleosynthesis models if the ejecta are clumpy with some admixture of $\mathrm{H}$ in the $\mathrm{Fe}$.

\section{MCSNR J0527-7104}

- The detected X-ray emission from MCSNR J0527-7104 was also found to be dominated by $\mathrm{Fe} \mathrm{L}$-shell emission.
However, unlike MCSNR J0506-7025 no soft X-ray shell was detected. The X-ray morphology of the Fe-rich ejecta was extremely unusual, extended to the north beyond the apparent optical shell. The $24 \mu \mathrm{m}$ and H I data indicate a low density cavity in the ambient medium to the north of the optical shell and we suggested that the blast wave has broken out into this cavity, allowing the shock heated ejecta to escape.

- We calculated the mass of Fe contained in the ejecta, which is also consistent with expected yields from Type Ia explosions if the ejecta are clumpy with some admixture of $\mathrm{H}$ in the $\mathrm{Fe}$.

MCSNR J0506-7025 and MCSNR J0527-7104 are new members of the recently identified class of evolved SNRs with centrally-peaked, Fe L-shell dominated emission (Borkowski et al. 2006), bringing the known population of such remnants in the LMC to 11. The overwhelming dominance of Fe emission lines in their spectra is evidence that they result from Type Ia explosions. The Fe-rich plasmas were found to be in collisional ionisation equilibrium, consistent with other members of the class and suggests that MCSNR J0506-7025 and MCSNR J0527-7104 most likely resulted from the "prompt" channel of single degenerate systems. The Fe cores of both remnants, in particular MCSNR J0527-7104, we found to be elongated/irregular, which appears to be a relatively common feature of this class of evolved SNRs.

Acknowledgements. We would like to thank our referee Fred Seward for reviewing our manuscript and for his suggestions to improve the paper. This research is funded by the Bundesministerium für Wirtschaft und Technologie/Deutsches Zentrum für Luft- und Raumfahrt (BMWi/DLR) through grant FKZ 50 OR 1309. M.S. acknowledges support by the Deutsche Forschungsgemeinschaft through the Emmy Noether Research Grant SA2131/1-1. P. M. acknowledges support by the Centre National d'Études Spatiales (CNES). E.T.W. acknowledges support by the Deutsche Forschungsgemeinschaft through the Research Grant WH-172/1-1. Cerro Tololo Inter-American Observatory (CTIO) is operated by the Association of Universities for Research in Astronomy Inc. (AURA), under a cooperative agreement with the National Science Foundation (NSF) as part of the National Optical Astronomy Observatories (NOAO). We gratefully acknowledge the support of CTIO and all the assistance that was provided for upgrading the Curtis Schmidt telescope. The MCELS project has been supported in part by NSF grants AST-9540747 and AST-0307613, and through the generous support of the Dean B. McLaughlin Fund at the University of Michigan, a bequest from the family of Dr. Dean B. McLaughlin in memory of his lasting impact on Astronomy. We used the karma software package developed by the ATNF. The Australia Telescope Compact Array is part of the Australia Telescope, which is funded by the Commonwealth of Australia for operation as a National Facility managed by CSIRO.

\section{References}

Arnaud, K. A. 1996, in Astronomical Data Analysis Software and Systems V, eds. G. H. Jacoby, \& J. Barnes, ASP Conf. Ser., 101, 17

Aubourg, É., Tojeiro, R., Jimenez, R., et al. 2008, A\&A, 492, 631

Badenes, C., Bravo, E., Borkowski, K. J., \& Domínguez, I. 2003, ApJ, 593, 358

Badenes, C., Borkowski, K. J., \& Bravo, E. 2005, ApJ, 624, 198

Balucinska-Church, M., \& McCammon, D. 1992, ApJ, 400, 699

Borkowski, K. J., Lyerly, W. J., \& Reynolds, S. P. 2001, ApJ, 548, 820

Borkowski, K. J., Hendrick, S. P., \& Reynolds, S. P. 2006, ApJ, 652, 1259

Bozzetto, L. M., \& Filipović, M. D. 2014, Ap\&SS, 351, 207

Bozzetto, L. M., Filipovic, M. D., Urosevic, D., \& Crawford, E. J. 2012, Serb. Astron. J., 185, 25

Bozzetto, L. M., Filipović, M. D., Crawford, E. J., et al. 2013, MNRAS, 432, 2177

Bozzetto, L. M., Filipović, M. D., Urošević, D., Kothes, R., \& Crawford, E. J. 2014a, MNRAS, 440, 3220

Bozzetto, L. M., Kavanagh, P. J., Maggi, P., et al. 2014b, MNRAS, 439, 1110

Cappellari, M., \& Copin, Y. 2003, MNRAS, 342, 345

Chen, L.-W., Fabian, A. C., \& Gendreau, K. C. 1997, MNRAS, 285, 449

Costain, C. H. 1960, MNRAS, 120, 248

Crawford, E. J., Filipovic, M. D., de Horta, A. Y., Stootman, F. H., \& Payne, J. L. 2008, Serbian Astron. J., 177, 61 
Davies, R. D., Elliott, K. H., \& Meaburn, J. 1976, Mem. R. Astron. Soc., 81, 89

Dickel, J. R., McIntyre, V. J., Gruendl, R. A., \& Milne, D. K. 2010, AJ, 140, 1567

Dickey, J. M., \& Lockman, F. J. 1990, ARA\&A, 28, 215

Diehl, S., \& Statler, T. S. 2006, MNRAS, 368, 497

Dwarkadas, V. V., \& Chevalier, R. A. 1998, ApJ, 497, 807

Fazio, G. G., Hora, J. L., Allen, L. E., et al. 2004, ApJS, 154, 10

Fesen, R. A., Blair, W. P., \& Kirshner, R. P. 1985, ApJ, 292, 29

Filipovic, M. D., Pietsch, W., Haynes, R. F., et al. 1998, A\&AS, 127, 119

Gooch, R. 1995, in Astronomical Data Analysis Software and Systems IV, eds.

R. A. Shaw, H. E. Payne, \& J. J. E. Hayes, ASP Conf. Ser., 77, 144

Haberl, F., \& Pietsch, W. 1999, A\&AS, 139, 277

Hamilton, A. J. S., Sarazin, C. L., \& Chevalier, R. A. 1983, ApJS, 51, 115

Hendrick, S. P., Borkowski, K. J., \& Reynolds, S. P. 2003, ApJ, 593, 370

Henize, K. G. 1956, ApJS, 2, 315

Hughes, J. P., Ghavamian, P., Rakowski, C. E., \& Slane, P. O. 2003, ApJ, 582, L95

Hughes, A., Staveley-Smith, L., Kim, S., Wolleben, M., \& Filipović, M. 2007, MNRAS, 382, 543

Iwamoto, K., Brachwitz, F., Nomoto, K., et al. 1999, ApJS, 125, 439

Jansen, F., Lumb, D., Altieri, B., et al. 2001, A\&A, 365, L1

Kahn, F. D. 1975, Int. Cosmic Ray Conf., 11, 3566

Kavanagh, P. J., Sasaki, M., \& Points, S. D. 2012, A\&A, 547, A19

Kavanagh, P. J., Sasaki, M., Points, S. D., et al. 2013, A\&A, 549, A99

Kavanagh, P. J., Sasaki, M., Whelan, E. T., et al. 2015, A\&A, 579, A63

Kim, S., Dopita, M. A., Staveley-Smith, L., \& Bessell, M. S. 1999, AJ, 118, 2797

Kim, S., Staveley-Smith, L., Dopita, M. A., et al. 2003, ApJS, 148, 473

Kosenko, D., Helder, E. A., \& Vink, J. 2010, A\&A, 519, A11

Kuntz, K. D., \& Snowden, S. L. 2008, A\&A, 478, 575

Kuntz, K. D., \& Snowden, S. L. 2010, ApJS, 188, 46

Maeda, K. 2012, in Death of Massive Stars: Supernovae and Gamma-Ray Bursts, IAU Symp., 279, 261

Maggi, P., Haberl, F., Kavanagh, P. J., et al. 2014, A\&A, 561, A76

Maggi, P., Haberl, F., Kavanagh, P. J., et al. 2016, A\&A, 585, A162
Mannucci, F., Della Valle, M., \& Panagia, N. 2006, MNRAS, 370, 773

Maoz, D. 2008, MNRAS, 384, 267

Mathewson, D. S., \& Clarke, J. N. 1973, ApJ, 179, 89

Meixner, M., Gordon, K. D., Indebetouw, R., et al. 2006, AJ, 132, 2268

Mills, B. Y., Turtle, A. J., Little, A. G., \& Durdin, J. M. 1984, Australian J. Phys., 37, 321

Murphy Williams, R., Chu, Y.-H., Dickel, J. R., et al. 1999, ApJ, 514, 798

Nishiuchi, M., Yokogawa, J., Koyama, K., \& Hughes, J. P. 2001, PASJ, 53, 99

Payne, J. L., White, G. L., \& Filipović, M. D. 2008, MNRAS, 383, 1175

Rieke, G. H., Young, E. T., Engelbracht, C. W., et al. 2004, ApJS, 154, 25

Sasaki, M., Plucinsky, P. P., Gaetz, T. J., et al. 2004, ApJ, 617, 322

Sault, R. J., Teuben, P. J., \& Wright, M. C. H. 1995, in Astronomical Data Analysis Software and Systems IV, eds. R. A. Shaw, H. E. Payne, \& J. J. E. Hayes, ASP Conf. Ser., 77, 433

Seward, F. D., Williams, R. M., Chu, Y.-H., et al. 2006, ApJ, 640, 327

Shull, J. M., \& van Steenberg, M. 1982, ApJS, 48, 95

Sim, S. A., Röpke, F. K., Hillebrandt, W., et al. 2010, ApJ, 714, L52

Smith, R. K., \& Hughes, J. P. 2010, ApJ, 718, 583

Smith, R. C., Points, S. D., \& Winkler, F. 2006, NOAO Newsletter, 85, 6

Snowden, S. L., Mushotzky, R. F., Kuntz, K. D., \& Davis, D. S. 2008, A\&A, 478,615

Staveley-Smith, L., Kim, S., Calabretta, M. R., Haynes, R. F., \& Kesteven, M. J. 2003, MNRAS, 339, 87

Strüder, L., Briel, U., Dennerl, K., et al. 2001, A\&A, 365, L18

Sturm, R. K. N. 2012, Ph.D. Thesis, Fakultät für Physik, Technische Universität München, Germany

Sullivan, M., Le Borgne, D., Pritchet, C. J., et al. 2006, ApJ, 648, 868

Turner, M. J. L., Abbey, A., Arnaud, M., et al. 2001, A\&A, 365, L27

Turtle, A. J., Pugh, J. F., Kenderdine, S., \& Pauliny-Toth, I. I. K. 1962, MNRAS, 124,297

Vink, J. 2012, AARv, 20, 49

Werner, M. W., Roellig, T. L., Low, F. J., et al. 2004, ApJS, 154, 1

Williams, R. M., \& Chu, Y.-H. 2005, ApJ, 635, 1077

Wilms, J., Allen, A., \& McCray, R. 2000, ApJ, 542, 914

Woosley, S. E., \& Kasen, D. 2011, ApJ, 734, 38 